\title{
Matrix-valued Subdivision Schemes for Generating Surfaces with Extraordinary Vertices
}

\author{
Charles K. Chui, Q Qingtang Jiang \\ Department of Mathematics \& Computer Science \\ University of Missouri-St. Louis \\ St. Louis, MO 63121
}

\begin{abstract}
Subdivision templates of numerical values are replaced by templates of matrices in this paper to allow the introduction of shape control parameters for the feasibility of achieving desirable geometric shapes at those points on the subdivision surfaces that correspond to extraordinary control vertices. Formulation of the matrix-valued subdivision surface is derived. Based on refinable bivariate spline function vectors for matrix-valued subdivisions, the notion of characteristic map introduced by Reif is extended from (scalar) surface subdivisions to matrix-valued subdivisions. The $C^{1}$ - and $C^{k}$-continuity of Reif and Prautzsch for matrix-valued subdivisions are discussed. To illustrate the general theory, the smoothness of matrix-valued triangular subdivision schemes for extraordinary vertices with valences 3 and 4 is analyzed. The issue of effective choices of the shape control parameters will also be discussed in this paper.
\end{abstract}

Keywords: Matrix-valued surface subdivision, matrix-valued templates, surface shape control, extraordinary vertices, characteristic map, $C^{k}$-continuity

\section{Introduction}

To design, generate, and display surfaces in the three-dimensional space efficiently, subdivision schemes are formulated in terms of certain templates (coefficient stencils) of numerical values that are used as weights for taking weighted averages of certain given "old" vertices (or more precisely, points in the threedimensional space) to generate "new" vertices, and perhaps to move the positions of the old vertices as well; and thereby, yielding a higher resolution of a discrete approximation of the target (subdivision) surface for each application (to be called iteration) of the templates. These points, regardless of being the old or new ones, are called vertices, since a "connectivity rule" must be followed to identify triangles or (non-planar) quadrilaterals, with these points as vertices, in order to be able to apply the templates properly. The initial vertices are called "control vertices" and the initial mesh of triangles, or of (nonplanar) quadrilaterals, is called a "control net".

In surface subdivisions, all vertices, perhaps with the exception of only a few, are "regular vertices". For a triangular mesh, a vertex is called regular, if it has valence equal to 6 , meaning that it is connected to precisely 6 adjacent vertices. For a quadrilateral mesh, the valence of a regular vertex is 4 . On

*Supported by NSF Grant \#CCR-0098331, ARO Grant \#W911NF-04-1-0298, and DARPA/NGA Grant \# HM158205-2-0003. This author is also with Department of Statistics, Stanford University, Stanford, CA 94305. 

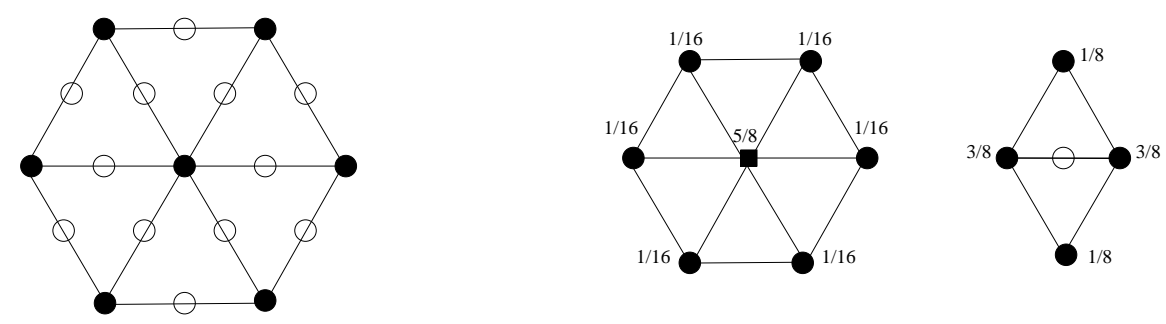

Figure 1: Subdivision templates of the Loop scheme for regular vertices (for moving old vertices and generating a new vertex corresponding to an edge point, respectively)
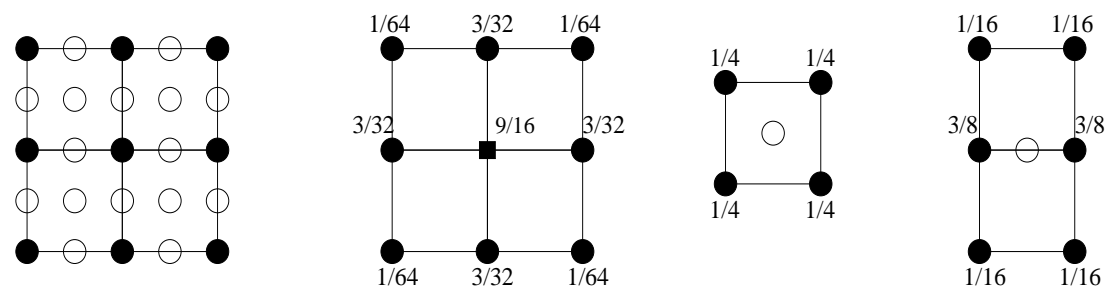

Figure 2: Subdivision templates of the Catmull-Clark scheme for regular vertices (for moving old vertices, generating a new vertex corresponding to a face point, and generating a new vertex corresponding to an edge point, respectively)

the other hand, subdivision templates are displayed in the two-dimensional space, along with certain triangles or quadrilaterals of regular shapes, in the so-called "parametric domain". Hence, the parametric representation of a triangular mesh, with regular vertices only, is a three-directional mesh; while that of a quadrilateral mesh, with regular vertices only, is a two-directional mesh, such as a rectangular grid. (See Fig. 1 for a typical example of templates along with the parametric domain for regular vertices of some triangular mesh, and Fig. 2 for an analogous example for a quadrilateral mesh.) Vertices that are not regular are called extraordinary vertices in the literature. In selecting a control net to generate some closed surface that is not topologically equivalent to a torus, extraordinary vertices are unavoidable; but fortunately, the number of them remains the same as that of the (initial) extraordinary control vertices, independent of the number of iterations being taken. Hence, extraordinary vertices are isolated in the iterative process of surface subdivision, and can be treated by applying certain specially designed local averaging rules that depend on the valences.

Templates for regular vertices are derived from the refinement equation (or two-scale relation) of some bivariate refinable function (or scaling function), with a finite refinement sequence (or two-scale coefficient sequence), to be called a "subdivision mask". For example, in the refinement equation

$$
\phi(\mathbf{x})=\sum_{\mathbf{k}} p_{\mathbf{k}} \phi(A \mathbf{x}-\mathbf{k}), \quad \mathbf{x} \in \mathbb{R}^{2},
$$

the function $\phi$ is a refinable function with (finite) subdivision mask $\left\{p_{\mathbf{k}}\right\}$ and dilation matrix $A$. It is clear that the subdivision mask sums to $|\operatorname{det}(A)|$ and that selection of the dilation matrix $A$ necessarily depends on the connectivity rule, which is commonly called "topological rule" in the literature. The most commonly used topological rule is the "1-to- 4 split" rule, that dictates the split of each triangle or rectangle in the parametric domain into four sub-triangles or sub-rectangles by connecting the midpoints of the edges, and thereby, introducing new vertices in the three-dimensional space, when the templates are applied to take weighted averages. Most of the well-known surface subdivision schemes 
such as the Catmull-Clark [2], Loop [25], and Butterfly [10] schemes engage the 1-to-4 split topological rule. For the 1-to- 4 split rule, the dilation matrix to be selected is simply $2 I_{2}$, both for triangular and quadrilateral meshes. Other topological rules of interest include the $\sqrt{3}[22,23,20,28,21,6]$ and the $\sqrt{2}$ split $[40,41,12,14,24]$ rules, with dilation matrices given, for example, by

$$
A_{1}=\left[\begin{array}{ll}
2 & -1 \\
1 & -2
\end{array}\right], \quad A_{2}=\left[\begin{array}{cc}
1 & 1 \\
1 & -1
\end{array}\right]
$$

respectively. We remark that these matrices are certainly not unique, and that while the 1-to- 4 split rule applies to both the triangular and quadrilateral meshes, the $\sqrt{3}$ rule applies only to the triangular mesh and the $\sqrt{2}$ rule to the quadrilateral mesh.

For a control net with control vertices $v_{\mathbf{k}}^{0}$, that are all regular, the refinement equation (1.1) immediately yields the "local averaging rule"

$$
v_{\mathbf{j}}^{m+1}=\sum_{\mathbf{k}} v_{\mathbf{k}}^{m} p_{\mathbf{j}-A \mathbf{k}}, \quad m=0,1, \cdots,
$$

where for each $m=1,2, \cdots, v_{\mathbf{k}}^{m}$ denote the set of vertices obtained after $m$ iterations; and for sufficiently large values of $m$, these vertices provide an accurate discrete approximation of the target subdivision surface, which is precisely given by the series representation

$$
f(\mathbf{x})=\sum_{\mathbf{k}} v_{\mathbf{k}}^{0} \phi(\mathbf{x}-\mathbf{k}), \quad \mathbf{x} \in \mathbb{R}^{2},
$$

with the control vertices $v_{\mathbf{k}}^{0}$ as coefficients. Also, the subdivision templates for rendering this surface can be easily formulated by applying (1.3). Hence, the order of smoothness of the target surface is determined by that of the refinable function $\phi$. If this refinable function is not a compactly supported piecewise polynomial with prescribed smoothness joining property (called a bivariate spline), the order of smoothness of $\phi$ can be analyzed by applying the theory of shift-invariant spaces $[3,8,15,19,17,27]$.

On the other hand, since custom-designed local weighted averaging rules are required to take care of the (isolated) extraordinary vertices, the order of smoothness at those points on the target surface that correspond to the extraordinary vertices no longer follows from that of $\phi$. The study of the order of smoothness and the development of algorithms to achieve the desired order of smoothness at such points on the subdivision surfaces constitute one of the most fundamental research problems on the subject of surface subdivisions. Among the many attempts to the study of the order of smoothness, Doo and Sabin [9] considered necessary conditions on the subdivision matrices, Ball and Storry [1] studied the continuity of moving tangent planes, and Reif [35] introduced the notion of "characteristic maps", formulating a $C^{1}$ continuity condition in terms of the regularity and injectivity of the characteristic maps and eigenvalues of the subdivision matrices. The $C^{1}$-continuity result was applied to analyze several interesting schemes in [37, 29, 30, 11, 42, 39]. Moreover, in the papers of Prautzsch [31] and of Zorin [43], the result of Reif [35] was extended to orders of smoothness higher than 1. Furthermore, Prautzsch and Umlauf [33, 34] constructed $C^{2}$ schemes near extraordinary vertices by modifying the Catmull-Clark and Loop schemes, but encountered the problem that the geometry at these points is undesirable, being practically flat. In addition, $C^{k}$ surfaces can be produced by Reif's TURBS scheme [36] and Prautzsch's freeform spline method [32], both based on spline patches.

In our recent work $[6,7]$, we introduced subdivision templates of matrices to gain certain desirable properties, such as shape control parameters, smaller template size, and interpolation. (See also [13] for 
the construction of Hermite interpolation schemes by the parametric approach.) In the present paper, we consider several issues related to the matrix-valued subdivision.

This paper is organized as follows. Recall that for control vertices $v_{\mathbf{k}}^{0}$ that are regular, the subdivision surface generated by any surface subdivision scheme, with subdivision templates of numerical values derived from (1.1), is precisely the surface with series representation given by (1.4). However, when templates of matrices are used, since the control vertices are enriched with shape control parameters as well, we need to understand what subdivision surfaces are to be generated. The answer to this question, along with a bivariate $C^{2}$ cubic spline demonstrative example and certain necessary preliminary results on matrix-valued subdivisions, will be discussed in Section 2. In Section 3, we discuss the results of Reif [35] and Prautzsch [31] on $C^{k}$-continuity of the matrix-valued subdivision surfaces near the extraordinary vertices. Two examples, based on the refinable bivariate $C^{2}$ cubic splines introduced in Section 2, will be given in Section 4 to illustrate the general result. In Section 5, we demonstrate the feasibility of some dramatic change in geometric shapes by adjusting the shape control parameters, and discuss the issue of certain appropriate choices of these parameters to achieve subdivision surfaces with desirable geometric shapes.

\section{Matrix-valued subdivisions}

Analogous to (scalar) subdivision schemes as discussed in the previous section, a matrix-valued (vector) subdivision scheme for regular vertices is also derived from some refinement equation

$$
\Phi(\mathbf{x})=\sum_{\mathbf{k} \in \mathbf{Z}^{2}} P_{\mathbf{k}} \Phi(A \mathbf{x}-\mathbf{k}), \quad \mathbf{x} \in \mathbb{R}^{2},
$$

but with matrix-valued refinement mask $\left\{P_{\mathbf{k}}\right\}$ for a suitable dilation matrix $A$, where $\Phi=\left[\phi_{0}, \cdots, \phi_{r-1}\right]^{T}$ is called a refinable (or scaling) function vector. For the refinable function vector to be useful for surface subdivisions in our discussion, its components $\phi_{k}, k=0, \cdots, r-1$, must be in $C^{2}$, have compact support with finite mask, and satisfy the condition of "generalized partition of unity":

$$
\sum_{\mathbf{k} \in \mathbf{Z}^{2}} \mathbf{w} \Phi(x-\mathbf{k}) \equiv 1, \quad \mathbf{x} \in \mathbb{R}^{2},
$$

for some constant $r$-vector $\mathbf{w}=\left[w_{0}, \cdots, w_{r-1}\right]$. By changing the order of the $\phi_{k} \mathrm{~s}$ and multiplying them with some constant, if necessary, we may, and will, assume that

$$
w_{0}=1 .
$$

Corresponding to the refinement equation (2.1), the local averaging rule, from which the subdivision template (of matrices) follows immediately, is given by

$$
\mathbf{v}_{\mathbf{k}}^{m+1}=\sum_{\mathbf{j}} \mathbf{v}_{\mathbf{j}}^{m} P_{\mathbf{k}-A \mathbf{j}}, \quad m=0,1, \cdots,
$$

where

$$
\mathbf{v}_{\mathbf{j}}^{m}:=\left[v_{\mathbf{j}}^{m}, s_{\mathbf{j}, 1}^{m} \cdots, s_{\mathbf{j}, r-1}^{m}\right]
$$

are "row-vectors" (and more precisely, $3 \times r$ matrices) with $r$ components of points $v_{\mathbf{j}}^{m}, s_{\mathbf{j}, \ell}^{m}, \ell=1, \cdots, r-1$, in $\mathbb{R}^{3}$. We will call the initial row vectors $\mathbf{v}_{\mathbf{j}}^{0}$, "control vectors", their first components $v_{\mathbf{j}}^{0}$, "control vertices", and the other components $s_{\mathbf{j}, 1}^{0}, \cdots, s_{\mathbf{j}, r-1}^{0}$, "shape control parameters". Of course, the assumption 
(2.3) is essential for the first components to be called control vertices as in the scalar subdivision consideration.

In the vector setting, for sufficiently large values of $m$, the vertices $v_{\mathbf{j}}^{m}$ provide an accurate discrete approximation of the target subdivision surface, which is precisely given by the series representation

$$
F(\mathbf{x})=\sum_{\mathbf{k}} v_{\mathbf{j}}^{0} \phi_{0}(\mathbf{x}-\mathbf{j})+\sum_{\mathbf{k}}\left(s_{\mathbf{j}, 1}^{0} \phi_{1}(\mathbf{x}-\mathbf{j})+\cdots+s_{\mathbf{j}, r-1}^{0} \phi_{r-1}(\mathbf{x}-\mathbf{j})\right)
$$

for $\mathbf{x}$ in the parametric domain, with the control vectors $\mathbf{v}_{\mathbf{j}}^{0}=\left[v_{\mathbf{j}}^{0}, s_{\mathbf{j}, 1}^{0}, \cdots, s_{\mathbf{j}, r-1}^{0}\right]$ as coefficients.

\subsection{Subdivision Surfaces}

In this subsection, we show that under certain appropriate conditions, the sequence of piecewise linear surfaces with vertices $v_{\mathbf{j}}^{m}$ generated by control vectors $\mathbf{v}_{\mathbf{j}}^{0}$ converges to the limit surface $F(\mathbf{x})$ given in (2.6). Our discussion follows from the relation between the subdivision algorithm and the cascade algorithm (see also the discussion in [27]). For simplicity, we only consider the dilation matrix $A=2 I_{2}$.

Let $\Phi=\left[\phi_{0}, \cdots, \phi_{r-1}\right]^{T}$ be a compactly supported refinable function vector in $L^{2}\left(\mathbb{R}^{2}\right)^{r}$ with dilation $A=2 I_{2}$, and $P=\left\{P_{\mathbf{k}}\right\}$ be the corresponding subdivision mask with $P_{\mathbf{k}}=\mathbf{0}, \mathbf{k} \notin[-N, N]^{2}$ for some $N \in \mathbb{N}$. Suppose $\Phi$ satisfies (2.2) for some constant vector $\mathbf{w}$ with the first component $w_{0}=1$.

Let $C_{P}$ be the cascade algorithm operator from $L^{2}\left(\mathbb{R}^{2}\right)^{r}$ to $L^{2}\left(\mathbb{R}^{2}\right)^{r}$ defined by

$$
C_{P} \Phi_{0}(\mathbf{x})=\sum_{\mathbf{k}} P_{\mathbf{k}} \Phi_{0}(2 \mathbf{x}-\mathbf{k})
$$

where $\Phi_{0}$ is a compactly supported function vector in $L^{2}\left(\mathbb{R}^{2}\right)^{r}$. Then $\left\{C_{P}^{m} \Phi_{0}\right\}_{m \in \mathbf{Z}_{+}}$is called a cascade algorithm sequence. To describe the convergence of this sequence, we consider

$$
T_{P}:=\left[B_{2 \mathbf{k}-\mathbf{j}}\right]_{\mathbf{k}, \mathbf{j} \in[-N, N]^{2}},
$$

where $B_{\mathbf{j}}:=\frac{1}{4} \sum_{\mathbf{k}} P_{\mathbf{k}-\mathbf{j}} \otimes \bar{P}_{\mathbf{k}}$, and $\otimes$ denotes the Kronecker product of two matrices $K$ and $L$, namely $K \otimes L=\left[k_{i j} L\right]$.

Proposition 1 Suppose that the subdivision mask $P=\left\{P_{\mathbf{k}}\right\}$ in (2.1) satisfies the property that 1 is a simple eigenvalue of $T_{P}$ and all other eigenvalue of $T_{P}$ lie in the open unit disk $|z|<1$. Also, assume that

$$
\sum_{\mathbf{k}} \mathbf{w} P_{2 \mathbf{k}}=\sum_{\mathbf{k}} \mathbf{w} P_{2 \mathbf{k}+(1,0)}=\sum_{\mathbf{k}} \mathbf{w} P_{2 \mathbf{k}+(0,1)}=\sum_{\mathbf{k}} \mathbf{w} P_{2 \mathbf{k}+(1,1)}=\mathbf{w}
$$

for some constant $r$-vector $\mathbf{w}=\left[1, w_{1}, \cdots, w_{r-1}\right]$. Let $\mathbf{v}_{\mathbf{j}}^{m}$ be the vectors defined by (2.4) for $A=2 I_{2}$ with (initial) control vectors $\mathbf{v}_{\mathbf{k}}^{0}, \mathbf{k} \in \mathbb{Z}^{2}$. Then the sequence of piecewise linear surfaces with vertices $v_{\mathbf{j}}^{m}$ converges (in the $L^{2}$-norm) to the limit surface $F(\mathbf{x})$ in (2.6).

Proof. For a compactly supported function vector $\Phi_{0}$ in $L^{2}\left(\mathbb{R}^{2}\right)^{r}$, it is easy to verify that

$$
\sum_{\mathbf{j}} \mathbf{v}_{\mathbf{j}}^{1} \Phi_{0}(2 \mathbf{x}-\mathbf{j})=\sum_{\mathbf{k}} \mathbf{v}_{\mathbf{k}}^{0}\left(C_{P} \Phi_{0}\right)(\mathbf{x}-\mathbf{k})
$$

and more general,

$$
\sum_{\mathbf{j}} \mathbf{v}_{\mathbf{j}}^{m} \Phi_{0}\left(2^{m} \mathbf{x}-\mathbf{j}\right)=\sum_{\mathbf{k}} \mathbf{v}_{\mathbf{k}}^{0}\left(C_{P}^{m} \Phi_{0}\right)(\mathbf{x}-\mathbf{k}), \quad m=1,2, \cdots
$$


Let $h(\mathbf{x})$ be the two dimensional "hat" function with $\sum_{\mathbf{k}} h(\mathbf{x}-\mathbf{k})=1$. By choosing

$$
\Phi_{0}(\mathbf{x})=h(\mathbf{x})[1,0, \cdots, 0]^{T},
$$

the left-hand side of (2.9) becomes $\sum_{\mathbf{j}} v_{\mathbf{j}}^{m} h\left(2^{m} \mathbf{x}-\mathbf{j}\right)$, which is the piecewise linear surface with vertices at $v_{\mathbf{j}}^{m}$. On the other hand, for $\Phi_{0}$ given in $(2.10)$, we have

$$
\sum_{\mathbf{k}} \mathbf{w} \Phi_{0}(\mathbf{x}-\mathbf{k})=\sum_{\mathbf{k}} \mathbf{w}[1,0, \cdots, 0] h(\mathbf{x}-\mathbf{k})=w_{0} \sum_{\mathbf{k}} h(\mathbf{x}-\mathbf{k})=1 .
$$

This property of $\Phi_{0}$, together with the assumptions stated in Proposition 1 , implies that $C_{P}^{m} \Phi_{0} \rightarrow \Phi$ in the $L^{2}\left(\mathbb{R}^{2}\right)^{r}$-norm as $m \rightarrow \infty$ (see [38], and for the convergence of vector subdivisions in general, see $[18,26,4])$. Therefore, the right-hand side of $(2.9)$ converges in the $L^{2}$-norm to $\sum_{\mathbf{k}} \mathbf{v}_{\mathbf{k}}^{0} \Phi(\mathbf{x}-\mathbf{k})$, which is the surface $F(\mathbf{x})$ given in $(2.6)$. $\diamond$

One may wonder what the limit surface would be if we use other components $s_{\mathbf{j}, \ell}^{m}, \ell=1, \cdots, r-1$, of $\mathbf{v}_{\mathbf{j}}^{m}$ as the vertices. For this more general consideration, we may consider a linear combination of the components of $\mathbf{v}_{\mathbf{j}}^{m}$ as the vertices. Let $C_{0}=\left[c_{0}, c_{1}, \cdots, c_{r-1}\right]^{T}$ be a nonzero constant vector, and consider the sequence of piecewise linear surfaces with vertices $\mathbf{v}_{\mathbf{j}}^{m} C_{0}, \mathbf{j} \in \mathbb{Z}^{2}, m=0,1, \cdots$. Let $\Phi_{1}(\mathbf{x}):=h(\mathbf{x}) C_{0}$, where $h(\mathbf{x})$ is the "hat" function considered above. Then $\sum_{\mathbf{j}} \mathbf{v}_{\mathbf{j}}^{m} \Phi_{1}\left(2^{m} \mathbf{x}-\mathbf{j}\right)$ represents the piecewise linear surface with vertices $\mathbf{v}_{\mathbf{j}}^{m} C_{0}, \mathbf{j} \in \mathbb{Z}^{2}$. Again, we have (2.9), with $\Phi_{0}$ replaced by $\Phi_{1}$. Observe that for this particular function vector $\Phi_{1}=h(\mathbf{x}) C_{0}$, we have

$$
\sum_{\mathbf{k}} \mathbf{w} \Phi_{1}(\mathbf{x}-\mathbf{k})=\sum_{\mathbf{k}} \mathbf{w} C_{0} h(\mathbf{x}-\mathbf{k})=\mathbf{w} C_{0} \sum_{\mathbf{k}} h(\mathbf{x}-\mathbf{k})=\mathbf{w} C_{0} .
$$

Under the assumptions stated in Proposition 1 , we see that $C_{P}^{m} \Phi_{1} \rightarrow\left(\mathbf{w} C_{0}\right) \Phi$ in the $L^{2}\left(\mathbb{R}^{2}\right)^{r}$-norm as $m \rightarrow \infty$. Thus the sequence of piecewise linear surfaces with vertices $\mathbf{v}_{\mathbf{j}}^{m} C_{0}$ converges (in $L^{2}$-norm) to the limit surface $\left(\mathbf{w} C_{0}\right) F(\mathbf{x})$, where $F(\mathbf{x})$ is given in (2.6). Therefore, if $\mathbf{w} C_{0}=0$, then the (limiting) subdivision surface is degenerate (i.e. equal to 0 ).

In particular, if the unit coordinate vectors $e_{i}:=[0, \cdots, 0,1,0, \cdots, 0], i=0, \cdots, r-1$, are used for $C_{0}$, meaning that the $(i+1)^{t h}$ components of $\mathbf{v}_{\mathbf{j}}^{m}, m=1,2, \cdots$, are used as the vertices, then the subdivision surfaces are $w_{i} F(\mathbf{x})$, respectively. Hence, if $w_{i}=0$, then we have the degenerate subdivision surface.

\subsection{Bivariate spline example}

In our recent work $[6,7]$, we have constructed refinable function vectors $\Phi$ with each component being a bivariate spline function (with small support) on the 6-directional mesh $\triangle^{3}$. More precisely, $\triangle^{3}$ is obtained by triangulation of the $x-y$ plane $\mathbb{R}^{2}$ with the grid lines $x=i, y=j, x-y=k, x+y=$ $\ell, x+2 y=m$, and $2 x+y=n$, where $i, j, k, \ell, m, n \in \mathbb{Z}$ (see a truncated portion shown on the left of Fig. 3). We remark that the reason for the choice of $\triangle^{3}$ here, as opposed to $\triangle_{-}^{3}=\left\{(x, y):(x,-y) \in \triangle^{3}\right\}$ in $[6,7]$, is to use the same domain of the characteristic map as that considered in [39]. For integers $d$ and $r$, with $0 \leq r<d$, let $S_{d}^{r}\left(\triangle^{3}\right)$ denote the collection of all (real-valued) functions in $C^{r}\left(\mathbb{R}^{2}\right)$ whose restrictions on each triangle of the triangulation $\triangle^{3}$ are bivariate polynomials of total degree $\leq d$. Each function $\phi$ in $S_{d}^{r}\left(\triangle^{3}\right)$ is called a bivariate $C^{r}$-spline of degree $d$ on $\triangle^{3}$. (See, for example, [5] for a general discussion.)

In $[6,7]$, we have introduced refinable function vectors for the spaces $S_{2}^{1}\left(\triangle^{3}\right), S_{3}^{2}\left(\triangle^{3}\right)$ and $S_{4}^{2}\left(\triangle^{3}\right)$. In particular, the refinable function vectors for $S_{2}^{1}\left(\triangle^{3}\right)$ and $S_{4}^{2}\left(\triangle^{3}\right)$ have the Hermite interpolating 

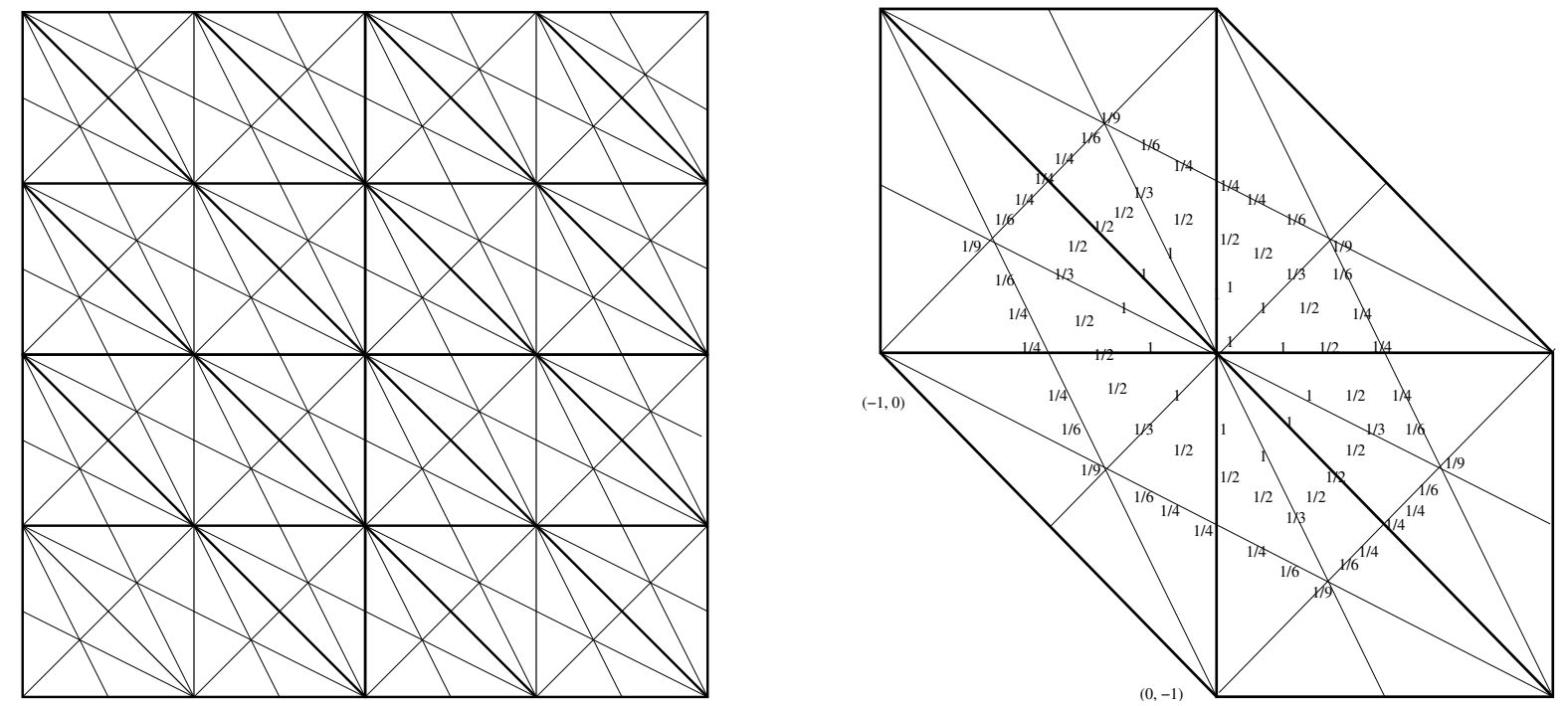

Figure 3: Six directional mesh $\triangle^{3}$ (on left), support and Bézier-nets of $\phi_{1}^{b}$ (on right)

properties of order 1 and 2, respectively (see [6] and [7] for the corresponding templates for Hermite interpolating matrix-valued subdivision schemes).

In [6], we have also constructed a basis function $\phi_{1}^{b} \in S_{3}^{2}\left(\triangle^{3}\right)$ with (minimum) support shown on the right of Fig. 3, where its nonzero Bézier coefficients are displayed. It is shown in [6] that with $A_{1}$ in (1.2) and

$$
\phi_{2}^{b}(\mathbf{x}):=\phi_{1}^{b}\left(\left(A_{1}^{-1}\right)^{T} \mathbf{x}\right)
$$

the function vector $\left[\phi_{1}^{b}, \phi_{2}^{b}\right]$ is refinable with the dilation matrix $2 I_{2}$, and its subdivision (or refinement) masks may be considered as an extension of the Loop scheme (where the mask of the quartic box spline $B_{222}$ on the three-directional mesh is used). In this paper, for the sake of better graphic display, we consider, instead, the refinable function vector

$$
\Phi^{b}:=\left[\phi_{2}^{b}, \frac{1}{4} \phi_{1}^{b}-\frac{1}{4} \phi_{2}^{b}\right]^{T}
$$

and its corresponding subdivision mask $\left\{P_{\mathbf{k}}\right\}$ with dilation matrix $2 I_{2}$. The nonzero matrices $P_{\mathbf{k}}$ are given by

$$
\begin{aligned}
& P_{0,0}=\frac{1}{8}\left[\begin{array}{cc}
6 & 4 \\
\frac{1}{2} & -2
\end{array}\right], \\
& P_{1,0}=P_{-1,0}=P_{0,1}=P_{0,-1}=P_{1,1}=P_{-1,-1}=B, \\
& P_{2,1}=P_{-2,-1}=P_{1,2}=P_{-1,-2}=P_{1,-1}=P_{-1,1}=C, \\
& P_{2,0}=P_{-2,0}=P_{0,2}=P_{0,-2}=P_{2,2}=P_{-2,-2}=D,
\end{aligned}
$$

where

$$
B=\frac{1}{24}\left[\begin{array}{cc}
11 & 8 \\
-2 & 1
\end{array}\right], \quad C=\frac{1}{24}\left[\begin{array}{cc}
4 & 4 \\
-1 & -1
\end{array}\right], \quad D=\frac{1}{24}\left[\begin{array}{cc}
1 & 4 \\
-\frac{1}{4} & -1
\end{array}\right] .
$$

The templates of the local averaging rule based on this subdivision mask for regular vertices are shown in Fig. 4. For convenience, we refer to this matrix-valued subdivision scheme as the $S_{3}^{2}$-subdivision. 

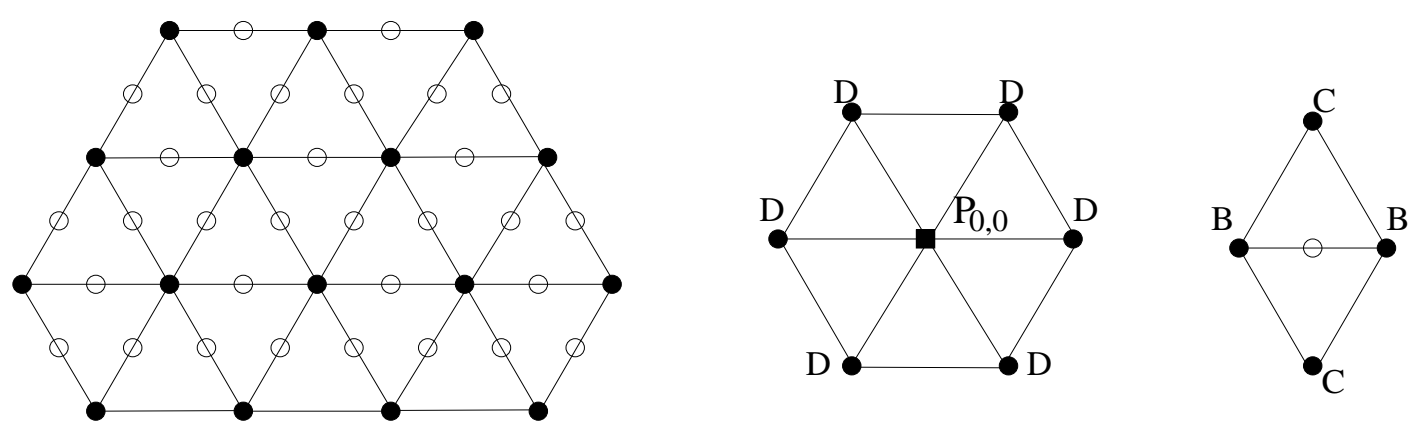

Figure 4: Templates for local averaging rule of $S_{3}^{2}$-subdivision for regular vertices

\section{Analysis of $C^{k}$-continuity for matrix-valued templates}

For the study of the smoothness property of the spline-based subdivision surfaces near extraordinary vertices, using matrix-valued templates, our key contribution is to understand how the matrix-valued (vector) subdivision generates the surfaces near extraordinary vertices. This allows us to treat splinebased vector subdivisions as a "special case" of spline-based scalar subdivisions for extraordinary vertices, as suggested by one of the reviewers. Let us first briefly summarize Reif's $C^{1}$-continuity and Prautzsch's $C^{k}$-continuity for scalar subdivision near the extraordinary vertices. Here and in the following, a $C^{k}$ surface is meant to be a $C^{k}$-manifold.

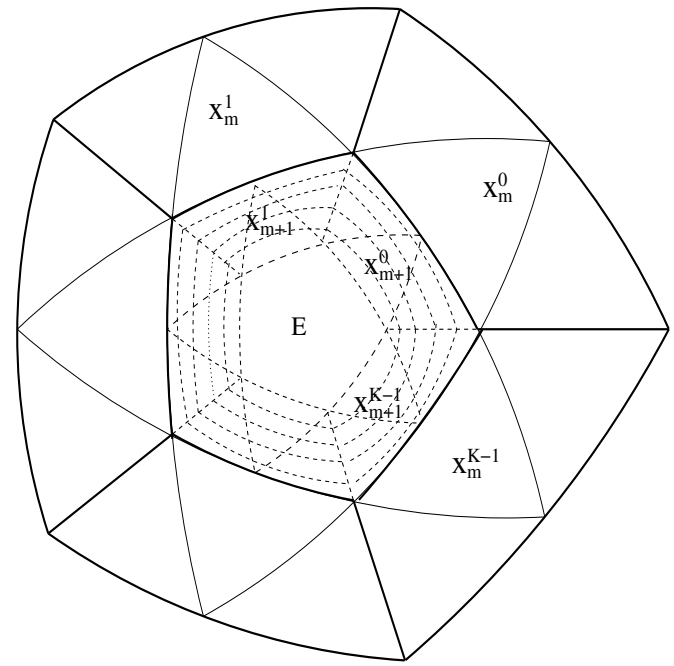

Figure 5: Layers $\mathbf{x}_{m}^{j}$

When a "generalized" $B$-spline subdivision is used to generate subdivision surfaces [35, 30], the standard spline subdivision is used for refinement for the regular vertices, whereas special averaging rules are used for the vertices near the extraordinary vertices (that is, valance $K \neq 6$ for triangular meshes and $K \neq 4$ for quadrilateral meshes). Since the subdivision masks considered here are of finite size and the extraordinary vertices are isolated in the iteration process of surface subdivisions, we will analyze meshes with a single extraordinary vertex. The spline surfaces $\mathbf{D}_{m}$ corresponding to the regular vertices after $m$ steps of subdivision iterations form an ascending nested sequence $\mathbf{D}_{0} \subset \mathbf{D}_{1} \subset \mathbf{D}_{2} \subset \ldots$ 
that converges to the limit surface $\mathbf{C}=\operatorname{clos}\left(\cup_{m \in \mathbf{z}_{+}} \mathbf{D}_{m}\right)$. With the prolongation of $\mathbf{D}_{m}$ defined by

$$
\mathbf{x}_{m}:=\cos \left(\mathbf{D}_{m+1} \backslash \mathbf{D}_{m}\right),
$$

the limit surface becomes $\mathbf{C}=\mathbf{D}_{0} \cup\left(\cup_{m \in \mathbf{Z}_{+}} \mathbf{x}_{m}\right)$. The sets $\mathbf{x}_{m}$ are ring-shaped surface layers which can be parameterized conveniently over a common domain $\Omega \times \mathbb{Z}_{K}, \mathbb{Z}_{K}:=\mathbb{Z} \bmod K$, consisting of $K$ copies of the compact set $\Omega$, where $\Omega$ is either

$$
\Omega^{\triangle}=\{(u, v) \mid u, v \geq 0,1 \leq u+v \leq 2\}
$$

for triangular meshes or

$$
\Omega^{\square}=\{(u, v) \mid 0 \leq u, v \leq 2\} \backslash\{(u, v) \mid 0 \leq u, v<1\}
$$

for quadrilateral meshes (see e.g., [39]). See Fig. 5 for the surface layers $\mathbf{x}_{m}^{j}$ for triangular meshes, and see Fig. 6 for the domains $\Omega^{\triangle}, \Omega^{\square}$.
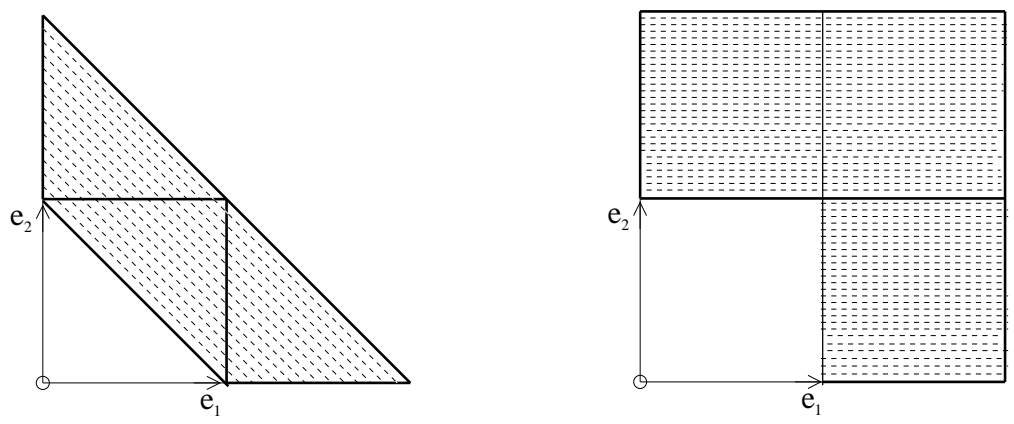

Figure 6: Domains $\Omega^{\triangle}$ (on left) and $\Omega^{\square}$ (on right)

Each surface layer $\mathbf{x}_{m}$ can be parameterized in terms of vertices $B_{m}^{\ell} \in \mathbb{R}^{3}$ and piecewise polynomial functions $N^{\ell}(u, v, j)$ according to

$$
\mathbf{x}_{m}: \Omega \times \mathbb{Z}_{K} \ni(u, v, j) \rightarrow \mathbf{x}_{m}(u, v, j)=\sum_{\ell=0}^{L} B_{m}^{\ell} N^{\ell}(u, v, j),
$$

where $L$ is a positive integer and $N^{\ell}(u, v, j)$ are spline functions that possess the property of partition of unity:

$$
\sum_{\ell=0}^{L} N^{\ell}(u, v, j) \equiv 1, \quad(u, v) \in \Omega, j \in \mathbb{Z}_{K} .
$$

For simplicity, we will use $\mathbf{x}_{m}^{j}(u, v)$ to denote $\mathbf{x}_{m}(u, v, j)$. Also, set

$$
N(u, v, j):=\left[N^{0}(u, v, j), N^{1}(u, v, j), \cdots, N^{L}(u, v, j)\right]^{T}, \quad B_{m}:=\left[B_{m}^{0}, B_{m}^{1}, \cdots, B_{m}^{L}\right] .
$$

Then we can write

$$
\mathbf{x}_{m}^{j}(u, v)=\mathbf{x}_{m}(u, v, j)=B_{m} N(u, v, j) .
$$

The subdivision scheme is determined by the so-called subdivision matrix $S$, where

$$
B_{m+1}=B_{m} S
$$


To explain Reif's result in [35], let the eigenvalues of $S$ be $\lambda_{0}, \lambda_{1}, \cdots$, arranged in non-increasing order of the absolute values $\left|\lambda_{i}\right|$. A sufficient condition for the convergence of subdivision is that $\lambda_{0}=1$ and $\left|\lambda_{1}\right|<1$. Reif [35] assumes that $\lambda_{1}=\lambda_{2}, \lambda_{1}$ has the geometric multiplicity 2, and considers the (left) row eigenvectors $\mathbf{u}^{1}=\left[u_{0}^{1}, \cdots, u_{L}^{1}\right], \mathbf{u}^{2}=\left[u_{0}^{2}, \cdots, u_{L}^{2}\right]$ associated with $\lambda_{1}$ and $\lambda_{2}$, respectively. Define the function vector

$$
m(u, v, j):=\left[\mathbf{u}^{1} N(u, v, j), \mathbf{u}^{2} N(u, v, j)\right]: \quad \Omega \times \mathbb{Z}_{K} \rightarrow \mathbb{R}^{2} .
$$

Here, $m(u, v, j)$ is called the characteristic map of $S$. In [35], Reif proved that if

$$
\left|\lambda_{3}\right|<\left|\lambda_{1}\right|
$$

and that the characteristic map $m(u, v, j)$ is regular and without self-intersections (where regularity means that the Jacobian of $m(u, v, j)$ with respect to $u, v$ does not vanish in $\Omega$ ), then the subdivision surface near the limit point corresponidng to the extraordinary vertices is a $C^{1}$ surface for almost all choices of control nets.

To explain Prautzsch's result in [31] on $C^{k}$-continuity for some $k \in \mathbb{N}$, denote $(x, y):=m(u, v, j), j \in$ $\mathbb{Z}_{K}$. Here, for simplicity, we assume that the algebraic multiplicity and geometric multiplicity of possible eigenvalues $\lambda_{i}$ having the form $\lambda_{i}=\lambda_{1}^{\alpha_{1}} \lambda_{2}^{\alpha_{2}}$ with $\alpha_{1}+\alpha_{2} \leq k$, are the same. For $0 \leq \ell \leq k$, consider

$$
S_{\ell}:=\operatorname{span}\left\{x^{\beta_{1}} y^{\beta_{2}}: \beta_{1}+\beta_{2}=\ell, \beta_{1}, \beta_{2} \in \mathbb{Z}_{+}\right\}
$$

In [31], Prautzsch proved that the subdivision surface is in $C^{k}$ near the limit point corresponding to the extraordinary vertex for almost all choices of (initial) control nets provided that the characteristic map $m(u, v, j)$ is regular and without self-intersections, and that the eigenvalue $\lambda_{i}$ of $S$ satisfies either $\left|\lambda_{i}\right|<\left|\lambda_{1}\right|^{k}$ or that $\lambda_{i}$ has the form $\lambda_{i}=\lambda_{1}^{\alpha_{1}} \lambda_{2}^{\alpha_{2}}$ with $\alpha_{1}+\alpha_{2} \leq k$, and $\mathbf{u}_{i} N \in S_{\alpha_{1}+\alpha_{2}}$, where $\mathbf{u}_{i}$ is a left eigenvector associated with $\lambda_{i}$ and $N$ is the spline vector defined by (3.2).

For a vector subdivision, starting from some initial control vectors $\mathbf{v}_{\mathbf{j}}^{0}:=\left[v_{\mathbf{j}}^{0}, s_{\mathbf{j}, 1}^{0}, \cdots, s_{\mathbf{j}, r-1}^{0}\right]$ (where we mention again that each $\mathbf{v}_{\mathbf{j}}^{0}$ is a $3 \times r$ matrix), we have surface layers $\mathbf{x}_{m}^{j}$ from the regular parts of the spline surfaces. The surface layers $\mathbf{x}_{m}^{j}$ can be parameterized as

$$
\mathbf{x}_{m}: \Omega \times \mathbb{Z}_{K} \ni(u, v, j) \rightarrow \mathbf{x}_{m}(u, v, j)=\sum_{\ell=0}^{L} \mathbf{v}_{\ell}^{m} \mathbf{N}^{\ell}(u, v, j),
$$

where in this case, $\mathbf{v}_{\ell}^{m}$ are $3 \times r$ matrices

$$
\mathbf{v}_{\ell}^{m}=\left[v_{\ell}^{m}, s_{\ell, 1}^{m}, \cdots, s_{\ell, r-1}^{m}\right]
$$

and

$$
\mathbf{N}^{\ell}(u, v, j)=\left[N_{1}^{\ell}(u, v, j), N_{2}^{\ell}(u, v, j), \cdots, N_{r}^{\ell}(u, v, j)\right]^{T}
$$

are spline column vectors of length $r$ which satisfy

$$
\sum_{\ell=0}^{L} \mathbf{w} \mathbf{N}^{\ell}(u, v, j) \equiv 1, \quad(u, v) \in \Omega, j \in \mathbb{Z}_{K},
$$

where $\mathbf{w}$ is the vector in (2.2). These spline vectors should be smooth enough, namely, they are at least $C^{1}$ when we discuss $C^{1}$-continuity, and they are at least $C^{k}$ when we discuss $C^{k}$-continuity. Set

$$
\mathbf{V}^{m}:=\left[\mathbf{v}_{0}^{m}, \mathbf{v}_{1}^{m}, \cdots, \mathbf{v}_{L}^{m}\right]
$$


and note that $\mathbf{V}^{m}$ is a row vector of length $r(L+1)$. Then we have the vector subdivision matrix $\mathbf{S}$ determined by

$$
\mathbf{V}^{m+1}=\mathbf{V}^{m} \mathbf{S}
$$

In this case, $\mathbf{S}$ is a $r(L+1) \times r(L+1)$ matrix, or $(L+1) \times(L+1)$ block matrix of $r \times r$ matrix blocks.

On one hand, it is obvious that when $r=1$, the vector subdivision is reduced to the scalar subdivision. On the other hand, comparing (3.1) and (3.5), for the smoothness of subdivision surfaces, one finds that the vector subdivision could be considered as a "special" scalar subdivision in the sense that a vector subdivision surface with initial vectors $\mathbf{v}_{\ell}^{m}=\left[v_{\ell}^{m}, s_{\ell, 1}^{m}, \cdots, s_{\ell, r-1}^{m}\right]$, and spline column vectors $\mathbf{N}^{\ell}(u, v, j)=\left[N_{1}^{\ell}(u, v, j), \quad N_{2}^{\ell}(u, v, j), \cdots, N_{r}^{\ell}(u, v, j)\right]^{T}$ could be considered as a scalar subdivision surface with initial vertices $v_{\ell}^{0}$ as well as "vertices" $s_{\ell, 1}^{0}, \cdots, s_{\ell, r-1}^{0}$ and their corresponding spline systems $N_{1}^{\ell}(u, v, j)$ as well as $N_{2}^{\ell}(u, v, j), \cdots, N_{r}^{\ell}(u, v, j)$. Therefore, the existing theorems on the convergence and smoothness of the scalar subdivision algorithms can be applied to the vector setting. In the following, we reformulate these results in the vector setting.

Let the eigenvalues of the vector subdivision matrix $\mathbf{S}$ be $\lambda_{0}, \lambda_{1}, \cdots$, arranged in non-increasing order of their absolute values $\left|\lambda_{i}\right|$. Here, for simplicity, we consider again the case that the algebraic multiplicity and geometric multiplicity of the eigenvalues $\lambda_{i}$ that have the form $\lambda_{i}=\lambda_{1}^{\alpha_{1}} \lambda_{2}^{\alpha_{2}}, \alpha_{1}+\alpha_{2} \leq k$, are the same. Let $\mathbf{u}^{1}, \mathbf{u}^{2}$ be (linearly independent) left eigenvectors associated with $\lambda_{1}, \lambda_{2}$ of $\mathbf{S}$. Let $m(u, v, j)$ be the characteristic map of $\mathbf{S}$, namely,

$$
m(u, v, j)=\left[\mathbf{u}^{1} \mathbf{N}(u, v, j), \mathbf{u}^{2} \mathbf{N}(u, v, j)\right]: \quad \Omega \times \mathbb{Z}_{K} \rightarrow \mathbb{R}^{2},
$$

where in the vector setting,

$$
\mathbf{N}(u, v, j):=\left[\mathbf{N}^{0}(u, v, j)^{T}, \mathbf{N}^{1}(u, v, j)^{T}, \cdots, \mathbf{N}^{L}(u, v, j)^{T}\right]^{T} .
$$

Then one has the following theorems about the convergence and smoothness for vector subdivision. (Here we will say the subdivision algorithm converges if there is a unique point $p_{0}$ such that for any $(u, v) \in \Omega, j \in \mathbb{Z}_{K},\left\{\mathbf{x}_{m}^{j}\right\}_{m}$ defined by (3.5) satisfies $\lim _{m \rightarrow \infty} \mathbf{x}_{m}^{j}(u, v)=p_{0}$.)

Theorem 1 The subdivision algorithm described above converges, provided that $\lambda_{0}=1$ and $\left|\lambda_{1}\right|<1$.

Theorem 2 Suppose $\lambda_{0}=1$, and $\lambda_{1}=\lambda_{2}$ is a real eigenvalue with algebraic and geometric multiplicity 2. If $1>\left|\lambda_{1}\right|>\left|\lambda_{3}\right|$ and the characteristic map of $\mathbf{S}$ is regular, then the subdivision surface is tangentplane continuous (i.e., has continuous turning normal vectors) for almost all choices of (initial) control vectors.

Theorem 3 Let $k \in \mathbb{N}$. Suppose that $\lambda_{0}=1$, and $\lambda_{1}=\lambda_{2}$ is a real eigenvalue with algebraic and geometric multiplicities equal to 2 . Then the subdivision surface is a $C^{k}$-manifold near the limit point $p_{0}$ for almost all choices of (initial) control vectors, provided that the characteristic map of $\mathbf{S}$ is regular and injective, and that any eigenvalue $\lambda_{i}$ of $\mathbf{S}, i \geq 3$, satisfies one of the following two conditions:

(i) $\left|\lambda_{i}\right|<\left|\lambda_{1}\right|^{k}$.

(ii) $\lambda_{i}$ has the form $\lambda_{i}=\lambda_{1}^{\alpha_{1}} \lambda_{2}^{\alpha_{2}}, \alpha_{1}+\alpha_{2} \leq k, \lambda_{i}$ has the same algebraic and geometric multiplicities, such that $\mathbf{W}_{i} \mathbf{N} \in S_{\alpha_{1}+\alpha_{2}}$, where $\mathbf{W}_{i}$ is a left eigenvector of $\mathbf{S}$ associated with $\lambda_{i}, \mathbf{N}$ is the spline vector defined by (3.8), and $S_{\alpha_{1}+\alpha_{2}}$ is defined by (3.4). 
In particular, we have the following.

Corollary 1 Let $k \in \mathbb{N}$. Suppose $\lambda_{0}=1$, and $\lambda_{1}=\lambda_{2}$ is a real eigenvalue with algebraic and geometric multiplicity 2, such that the characteristic map of $\mathbf{S}$ is regular and injective. Then the subdivision surface is a $C^{k}$-manifold near the limit point $p_{0}$ for almost all choices of (initial) control vectors, provided that

$$
\left|\lambda_{3}\right|<\left|\lambda_{1}\right|^{k}
$$

As mentioned above, for the study of the surface smoothness conditions, the vector subdivision for extraordinary vertices could be considered as a special case of scalar subdivisions. Therefore, Theorems 1-3 follow from those in [35] and [31]. The reader is referred to an expanded version of this paper, downloadable at www.cs.umsl.edu/ jiang, for more details. One of the essential difference between scalar and vector subdivisions is the left eigenvectors of the subdivision matrices $S$ (for the scalar subdivision) and $\mathbf{S}$ (for the vector subdivision). For example, $[\mathbf{w}, \mathbf{w}, \cdots, \mathbf{w}]$, where $\mathbf{w}$ is the vector in (2.2), is the left eigenvector of $\mathbf{S}$ associated with $\lambda_{0}=1$, while in the scalar setting, $[1,1, \cdots, 1]$ is the left 1-eigenvector of $S$.

Remark 1 Let $\mathbf{u}^{1}, \mathbf{u}^{2}$ be (linearly independent) left eigenvectors of the vector subdivision matrix $\mathbf{S}$ associated with $\lambda_{1}, \lambda_{2}$, respectively. Note that $\mathbf{u}^{1}, \mathbf{u}^{2}$ are $r(L+1)$-vectors, namely,

$$
\mathbf{u}^{1}=\left[u_{1}^{1}, u_{2}^{1}, \cdots, u_{r(L+1)}^{1}\right], \quad \mathbf{u}^{2}=\left[u_{1}^{2}, u_{2}^{2}, \cdots, u_{r(L+1)}^{2}\right] .
$$

Corresponding to the subdivision mask of $r \times r$ matrices, we write

$$
\mathbf{U}^{1}=:\left[\mathbf{u}_{0}^{1}, \cdots, \mathbf{u}_{L}^{1}\right], \quad \mathbf{U}^{2}=:\left[\mathbf{u}_{0}^{2}, \cdots, \mathbf{u}_{L}^{2}\right]
$$

where

$$
\mathbf{u}_{\ell}^{1}:=\left[u_{\ell r+1}^{1}, u_{\ell r+2}^{1}, \cdots, u_{(\ell+1) r}^{1}\right], \quad \mathbf{u}_{\ell}^{2}:=\left[u_{\ell r+1}^{2}, u_{\ell r+2}^{2}, \cdots, u_{(\ell+1) r}^{2}\right], \quad \ell=0,1, \cdots, L .
$$

Then the characteristic map of $\mathbf{S}$ can be written as the function vector:

$$
m(u, v, j)=\left[\sum_{\ell=0}^{L} \mathbf{u}_{\ell}^{1} \mathbf{N}^{\ell}(u, v, j), \sum_{\ell=0}^{L} \mathbf{u}_{\ell}^{2} \mathbf{N}^{\ell}(u, v, j)\right]: \quad \Omega \times \mathbb{Z}_{K} \rightarrow \mathbb{R}^{2} .
$$

Based on this expression of the characteristic map for spline-based subdivisions in (3.10), when the refinable function vector $\Phi$ is not a spline vector, the characteristic map is defined as the function $M(u, v, j): \Omega \times \mathbb{Z}_{K} \rightarrow \mathbb{R}^{2}$ by applying the local averaging rules for the regular vertices to the particular control vectors

$$
\left[\begin{array}{l}
\mathbf{u}_{i}^{1} \\
\mathbf{u}_{i}^{2}
\end{array}\right] \in \mathbb{R}^{2 \times r}
$$

where $\left[\cdots, \mathbf{u}_{i}^{1}, \cdots\right]$ and $\left[\cdots, \mathbf{u}_{i}^{2}, \cdots\right]$ are (linearly independent) left eigenvectors associated with $\lambda_{1}$ and $\lambda_{2}$, respectively. The reader is referred to [42] for the definition of the characteristic map for the non-spline subdivisions in the scalar setting. In the vector setting, the definition of characteristic map is similar, by replacing the particular control vertices in $\mathbb{R}^{2}$ from the left eigenvectors associated with the eigenvalues $\lambda_{1}$ and $\lambda_{2}$ by the control vectors $\mathbf{u}_{i}^{1}, \mathbf{u}_{i}^{2}$ mentioned above. More precisely, with the assumption (2.3), we use the first components of $\mathbf{u}_{i, m}^{1}$ and $\mathbf{u}_{i, m}^{2}$ to define the characteristic map, where $\mathbf{u}_{i, m}^{1}$ and $\mathbf{u}_{i, m}^{2}$ are the vectors resulted from the $m$-th vector subdivision iterations with $\mathbf{u}_{i}^{1}$ and $\mathbf{u}_{i}^{2}$ as initial vectors, respectively. With this characteristic map for the vector subdivision, the above results (Theorems 2,3 and Corollary 1) will remain valid for the non-spline considerations. 


\section{Examples}

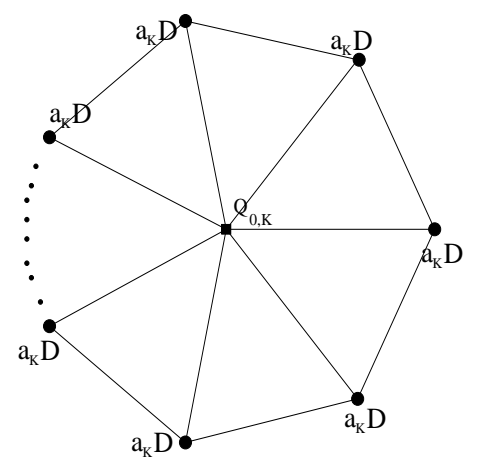

Figure 7: Template for local averaging rule of $S_{3}^{2}$-subdivision for extraordinary vertices

In this section, we use the $S_{3}^{2}$-subdivision as an example to illustrate the theorems developed in Section 3. First we recall that the $S_{3}^{2}$-subdivision already generates $C^{2}$ spline surfaces in the absence of extraordinary vertices. We therefore need to use special local averaging rules for the extraordinary vertices, so that the modified scheme generates at least $C^{1}$ surfaces in general. Let us consider the local averaging rule for the extraordinary vertices having valence $K \neq 6$ as shown in Fig. 7, for some constant $a_{K}$ and $2 \times 2$ matrix $Q_{0, K}$, which depend on the valence $K$, where $D$ is the matrix given in (2.12). Here we just consider the cases valences $K=3$ and $K=4$.

For the regularity and injectivity of the characteristic map $m(u, v, j)$ for the $S_{3}^{2}$-subdivision, by the symmetry of the subdivision schemes, we need only discuss those of $m(u, v, 0)=:\left[\psi_{1}(u, v), \psi_{2}(u, v)\right]$, which is further reduced to discussion of the positiveness of $\frac{\partial \psi_{1}}{\partial v}$ and $\frac{\partial \psi_{2}}{\partial v} \operatorname{since} \psi_{1}(u, v)=\psi_{1}(v, u), \psi_{2}(u, v)=$ $-\psi_{2}(v, u)$ (see $[30,39]$ for the detail discussions). We use the partial derivatives $\frac{\partial \phi_{1}^{b}}{\partial v}, \frac{\partial \phi_{2}^{b}}{\partial v}$ to evaluate $\frac{\partial \psi_{1}}{\partial v}$ and $\frac{\partial \psi_{2}}{\partial v}$. For this purpose, we have the relations

$$
\frac{\partial \phi_{1}^{b}}{\partial v}(u, v)=-6 \varphi_{2}(u, v)-12 \varphi_{3}(u, v), \quad \frac{\partial \phi_{2}^{b}}{\partial v}(u, v)=6 \varphi_{3}\left(\left(A_{1}^{-1}\right)^{T}(u, v)^{T}\right),
$$

where $\varphi_{2}, \varphi_{3}$ are splines in $S_{2}^{1}\left(\triangle^{3}\right)$ considered in [6], whose Bézier coefficients are displayed in Fig. 8.

For valance $K=4$, we choose

$$
a_{4}=\frac{1}{4}\left(9-8 x_{4}\right), \quad Q_{0,4}=\left[\begin{array}{cc}
x_{4} & \frac{1}{2} \\
\frac{23}{32}-\frac{3}{4} x_{4} & x_{4}-\frac{5}{8}
\end{array}\right],
$$

where $x_{4} \in \mathbb{R}$. The non-zero eigenvalues (algebraic multiplicities of eigenvalues being accounted individually) of the corresponding subdivision matrix $\mathbf{S}$ are

$$
1,3 / 8,3 / 8, x_{4}-1 / 2, x_{4}-1 / 2,1 / 8,1 / 8,1 / 8,1 / 8,1 / 8,1 / 8,1 / 8,1 / 8,1 / 8 .
$$

The left eigenvectors associated with the eigenvalues $3 / 8,3 / 8$ are independent of $x_{4}$. With the labelling of the indices in [42] (refer to Fig. 9 for $K=3$ ), two left eigenvectors associated with $3 / 8$ are given by

$$
\begin{aligned}
& u_{0}^{1}=[0,0], u_{1}^{1}=u_{2}^{1}=[9,9], u_{3}^{1}=u_{4}^{1}=-9[1,1], u_{5}^{1}=u_{6}^{1}=[95 / 4,23], \\
& u_{7}^{1}=u_{8}^{1}=-[95 / 4,23], u_{9}^{1}=27[1,1], u_{10}^{1}=[0,0], u_{11}^{1}=-27[1,1], \\
& u_{12}^{1}=[0,0], u_{13}^{1}=u_{14}^{1}=-u_{15}^{1}=-u_{16}^{1}=[503 / 12,125 / 3], u_{17}^{1}=47[1,1], u_{18}^{1}=14[1,1], \\
& u_{19}^{1}=-47[1,1], u_{20}^{1}=-14[1,1], u_{21}^{1}=47[1,1], u_{22}^{1}=-14[1,1], u_{23}^{1}=-47[1,1], u_{24}^{1}=14[1,1],
\end{aligned}
$$



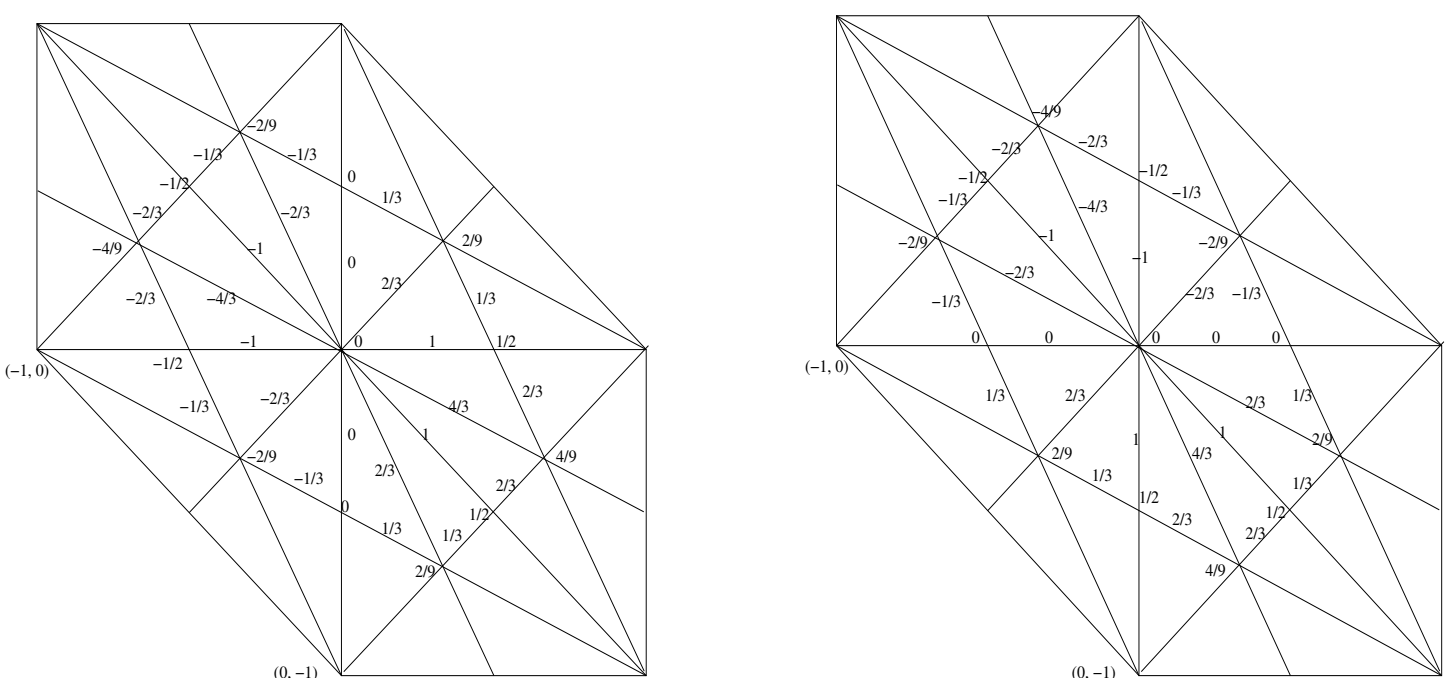

Figure 8: Bézier coefficients of $4 \varphi_{2}$ (on left) and $4 \varphi_{3}$ (on right)
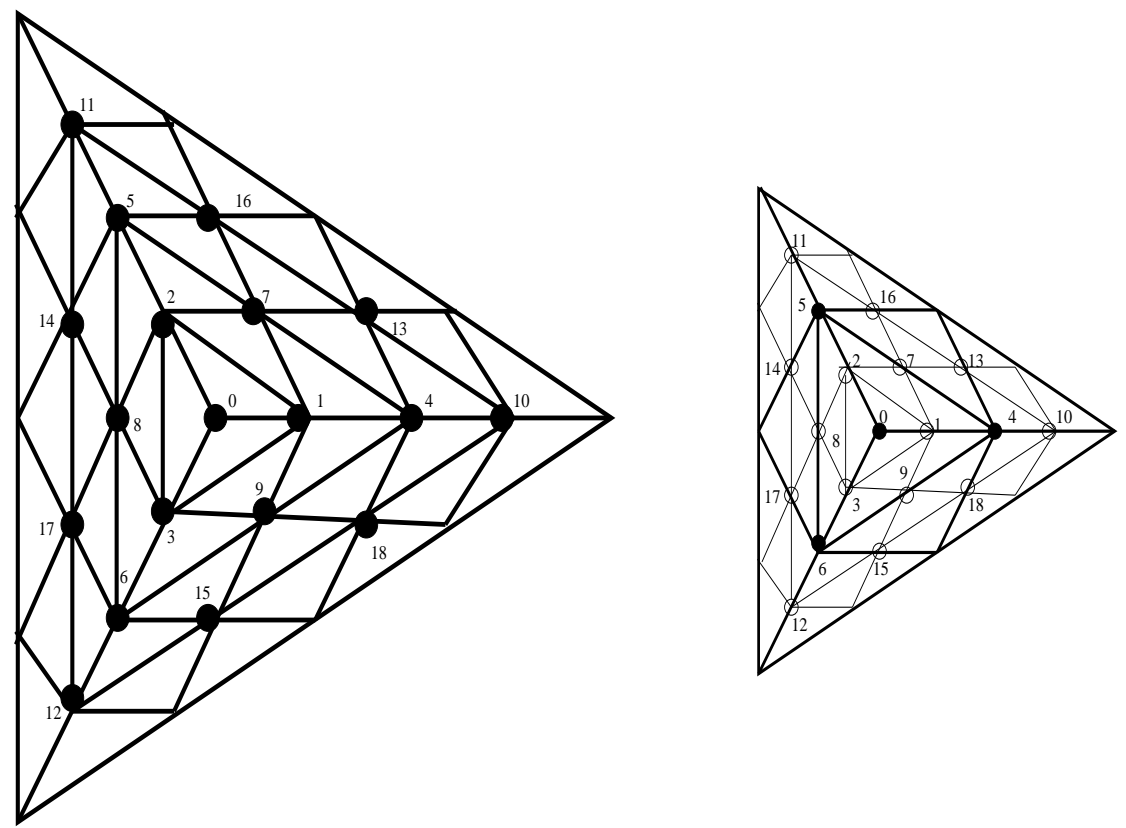

Figure 9: Labels of vertices near an extraordinary vertex: labels for old vertices (on left); labels for new vertices (on right)

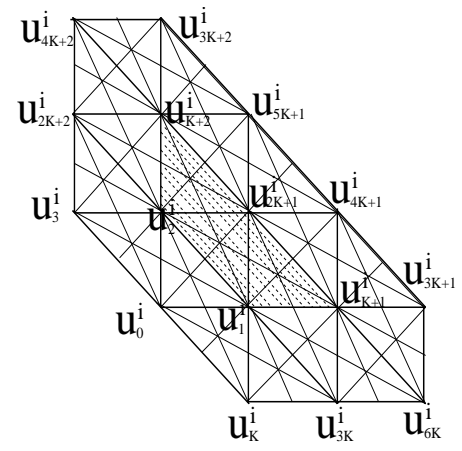

Figure 10: $\psi_{i}(u, v), i=1,2$, for $(u, v) \in \Omega^{\triangle}$ are determined by these vectors $\mathbf{u}_{\ell}^{i}$ for valence $=K$ case 

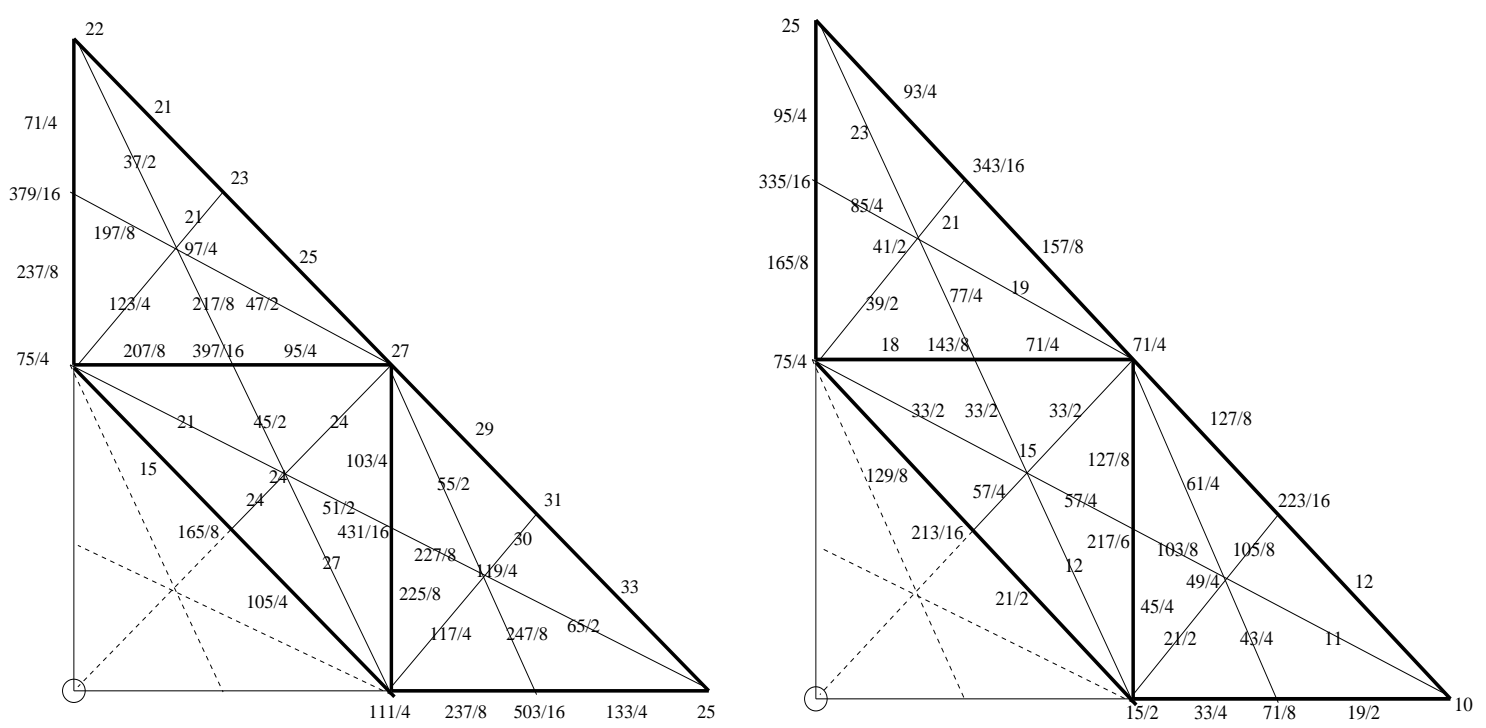

Figure 11: Bézier coefficients of $\frac{\partial \psi_{1}}{\partial v}$ (on left) and $\frac{\partial \psi_{2}}{\partial v}$ (on right) when $K=4$

and

$$
\begin{aligned}
& u_{0}^{2}=[0,0], u_{1}^{2}=-9[1,1], u_{2}^{2}=u_{3}^{2}=[9,9], u_{4}^{2}=-9[1,1], u_{5}^{2}=-u_{6}^{2}=-u_{7}^{2}=u_{8}^{2}=-[95 / 4,23] \\
& u_{9}^{2}=[0,0], u_{10}^{2}=27[1,1], u_{11}^{2}=[0,0], u_{12}^{2}=-27[1,1] \\
& u_{13}^{2}=-u_{14}^{2}=-u_{15}^{2}=u_{16}^{2}=[-503 / 12,-125 / 3], u_{17}^{2}=-14[1,1], u_{18}^{2}=47[1,1], \\
& u_{19}^{2}=14[1,1], u_{20}^{2}=-47[1,1], u_{21}^{2}=14[1,1], u_{22}^{2}=47[1,1], u_{23}^{2}=-14[1,1], u_{24}^{2}=-47[1,1] .
\end{aligned}
$$

The particular vectors $u_{\ell}^{i}$ that determine $\frac{\partial \psi_{1}}{\partial v}(u, v), \frac{\partial \psi_{2}}{\partial v}(u, v),(u, v) \in \Omega^{\triangle}$ are shown in Fig. 10. Based on these vectors and (4.1), we can calculate $\frac{\partial \psi_{1}}{\partial v}, \frac{\partial \psi_{2}}{\partial v}$ with Bézier coefficients shown in Fig. 11. Since all the Bézier coefficients are positive, we see that $\frac{\partial \psi_{1}}{\partial v}(u, v)>0, \frac{\partial \psi_{2}}{\partial v}(u, v)>0$ for $(u, v)$ on $\Omega^{\triangle}$. Thus, since

$$
\begin{gathered}
\triangle_{m}(u, v, 0)=\left|\begin{array}{cc}
\frac{\partial \psi_{1}}{\partial u}(u, v) & \frac{\partial \psi_{1}}{\partial v}(u, v) \\
\frac{\partial \psi_{2}}{\partial u}(u, v) & \frac{\partial \psi_{2}}{\partial v}(u, v)
\end{array}\right|=\left|\begin{array}{cc}
\frac{\partial \psi_{1}}{\partial v}(v, u) & \frac{\partial \psi_{1}}{\partial v}(u, v) \\
-\frac{\partial \psi_{2}}{\partial v}(v, u) & \frac{\partial \psi_{2}}{\partial v}(u, v)
\end{array}\right| \\
=\frac{\partial \psi_{1}}{\partial v}(v, u) \frac{\partial \psi_{2}}{\partial v}(u, v)+\frac{\partial \psi_{1}}{\partial v}(u, v) \frac{\partial \psi_{2}}{\partial v}(v, u)>0, \quad(u, v) \in \Omega^{\triangle}
\end{gathered}
$$

the characteristic map $m(u, v, 0)$ is regular, and it follows that $m(u, v, 0)$ is also injective by following the discussions in [30, 39]. So by Corollary 1 , for $\left|x_{4}-1 / 2\right|<3 / 8$, the limit surface is $C^{1}$. By Corollary 1 , for $\left|x_{4}-1 / 2\right|<(3 / 8)^{2}=9 / 64$, the limit surface is $C^{2}$. For example, let us choose $x_{4}=1 / 2+1 / 16=9 / 16$.

Let $\triangle_{0}$ be the octahedron with vertices

$$
v_{0}^{0}=[0,0,1]^{T}, v_{1}^{0}=[1,0,0]^{T}, v_{2}^{0}=[0,1,0]^{T}, v_{3}^{0}=[-1,0,0]^{T}, v_{4}^{0}=[0,-1,0]^{T}, v_{5}^{0}=[0,0,-1]^{T},
$$

as shown on the left of Fig. 12. We use these vertices as the first columns of the control vectors. If we choose the other columns of the control vectors, the shape control parameters, to be $s_{j, 1}^{0}=[0,0,0]^{T}, 0 \leq$ $j \leq 5$, we have $v_{j}^{1}, j=0,1, \cdots, 17$, by one $S_{3}^{2}$-subdivision iteration, as shown in the middle of Fig. 12 . The (limit) subdivision surface is displayed on the left of Fig. 12.

Zoom-in views near an extraordinary vertex $E$ for 4,5 , and 8 iteration steps, respectively, are shown in Fig. 13. It is clear that the $S_{3}^{2}$-subdivision scheme assures $C^{2}$-smoothness everywhere, with the possible 

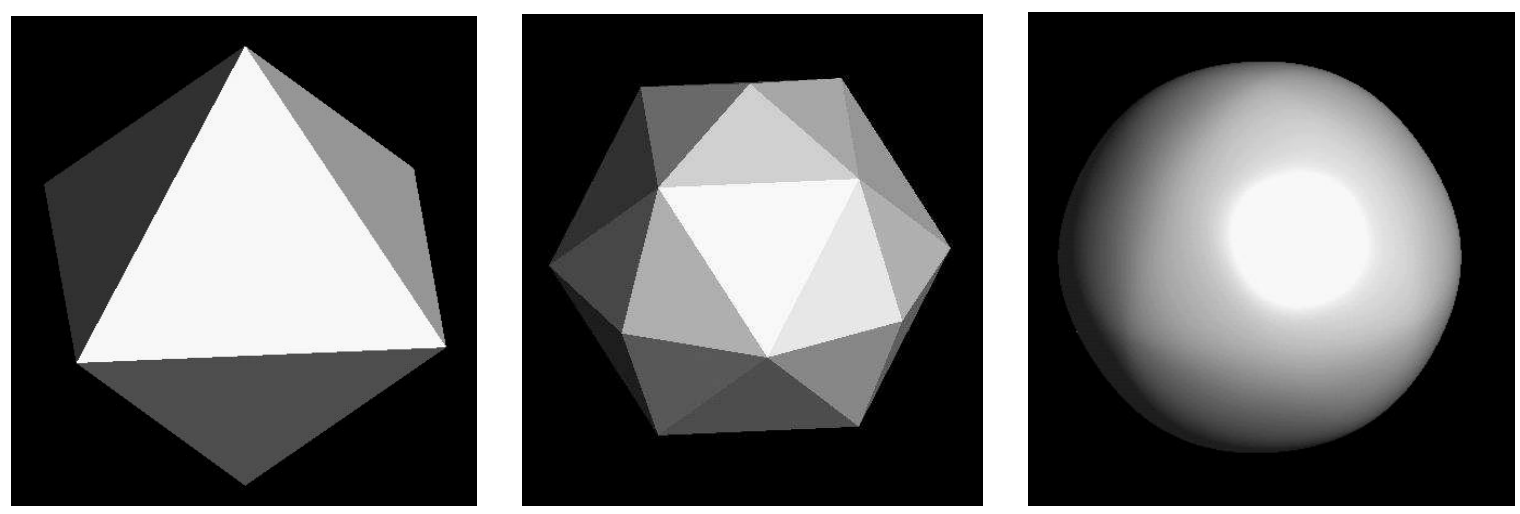

Figure 12: Initial closed surface (octahedron, on left), finer surface (in middle) after one iteration with shape control parameters $s_{j, 1}^{0}=[0,0,0]^{T}, 0 \leq j \leq 7$, and limit surface (on right), by $S_{3}^{2}$-subdivision with $x_{4}=9 / 16$
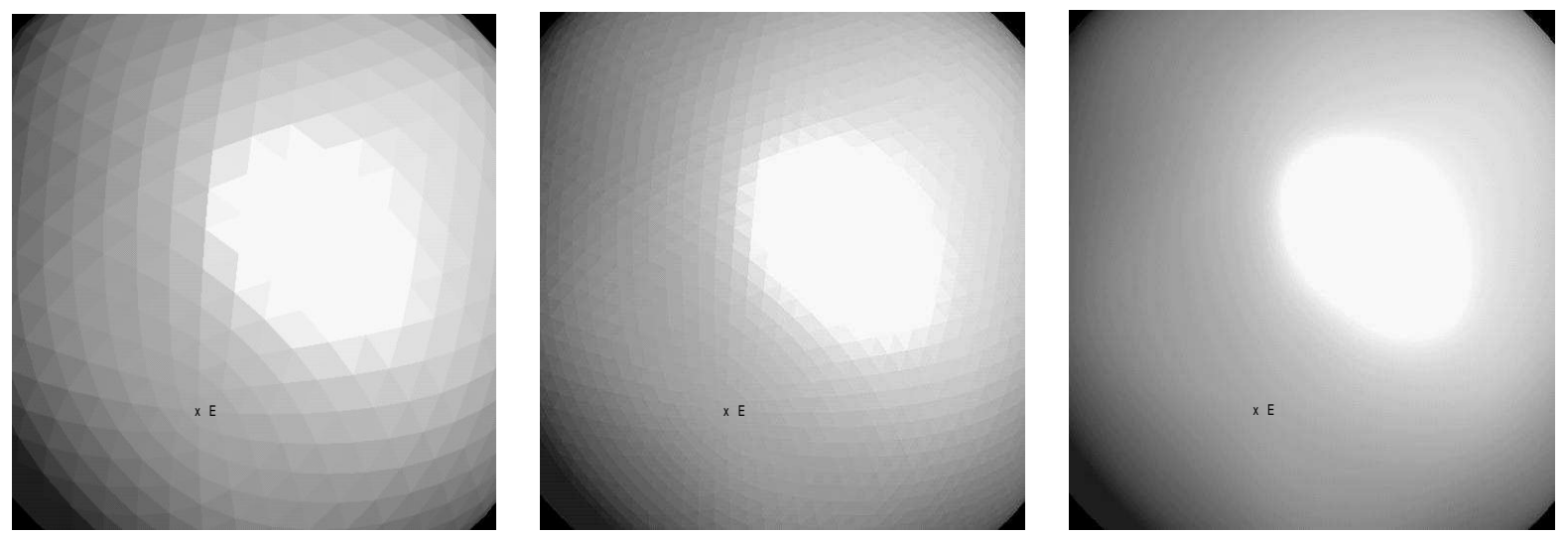

Figure 13: From left to right, finer surfaces after iteration steps $=4,5,8$, with octahedron as initial control mesh, by $S_{3}^{2}$-subdivision with $x_{4}=9 / 16$ 
exception of the extraordinary vertices. In Fig. 13, observe that the smoothness near the extraordinary vertex $E$ is even more visually pleasing than the $C^{2}$-smoothness of the remaining zoom-in surface, which confirms the result on

For valance $K=3$, we choose

$$
a_{3}=\frac{1}{3}\left(9-8 x_{3}\right), \quad Q_{0,3}=\left[\begin{array}{cc}
x_{3} & \frac{1}{2} \\
\frac{23}{32}-\frac{3}{4} x_{3} & x_{3}-\frac{5}{8}
\end{array}\right],
$$

where $x_{3} \in \mathbb{R}$. The non-zero eigenvalues of the corresponding subdivision matrix $\mathbf{S}$ are

$$
1,1 / 4,1 / 4, x_{3}-1 / 2, x_{3}-1 / 2,1 / 8,1 / 8,1 / 8,1 / 8,1 / 8,1 / 8
$$

The left eigenvectors associated with eigenvalues $1 / 4,1 / 4$ are independent of $x_{3}$. With the labelling of the indices used in [42] again as shown in Fig. 9, two left eigenvectors associated with $1 / 4$ are given by

$$
\begin{aligned}
& u_{0}^{1}=[0,0], u_{1}^{1}=2[1,1], u_{2}^{1}=2[1,1], u_{3}^{1}=-4[1,1], u_{4}^{1}=8[1,1], u_{5}^{1}=8[1,1], u_{6}^{1}=-16[1,1], \\
& u_{7}^{1}=12[1,1], u_{8}^{1}=-6[1,1], u_{9}^{1}=-6[1,1], u_{10}^{1}=18[1,1], u_{11}^{1}=18[1,1], u_{12}^{1}=-36[1,1], \\
& u_{13}^{1}=26[1,1], u_{14}^{1}=-4[1,1], u_{15}^{1}=-22[1,1], u_{16}^{1}=26[1,1], u_{17}^{1}=-22[1,1], u_{18}^{1}=-4[1,1],
\end{aligned}
$$

and

$$
\begin{aligned}
& u_{0}^{2}=[0,0], u_{1}^{2}=-2[1,1], u_{2}^{2}=2[1,1], u_{3}^{2}=[0,0], u_{4}^{2}=-8[1,1], u_{5}^{2}=8[1,1], u_{6}^{2}=[0,0], \\
& u_{7}^{2}=[0,0], u_{8}^{2}=6[1,1], u_{9}^{2}=-6[1,1], u_{10}^{2}=-18[1,1], u_{11}^{2}=18[1,1], u_{12}^{2}=[0,0], \\
& u_{13}^{2}=-6[1,1], u_{14}^{2}=16[1,1], u_{15}^{2}=-10[1,1], u_{16}^{2}=6[1,1], u_{17}^{2}=10[1,1], u_{18}^{2}=-16[1,1] .
\end{aligned}
$$

Based on these vectors and (4.1), we can calculate $\frac{\partial \psi_{1}}{\partial v}, \frac{\partial \psi_{2}}{\partial v}$ with Bézier coefficients shown in Fig. 14 . Since all Bézier coefficients are positive (except zeros on one boundary of $\Omega^{\triangle}$ ), we have that $\frac{\partial \psi_{1}}{\partial v}(u, v)>$ $0, \frac{\partial \psi_{2}}{\partial v}(u, v)>0$ for $(u, v)$ on $\Omega^{\triangle}$ (except $\frac{\partial \psi_{2}}{\partial v}(u, v)=0$ for $\left.v=0,1 \leq u \leq 2\right)$. Thus the characteristic map is regular and injective. So by Theorem 3, for $\left|x_{3}-1 / 2\right|<1 / 4$, the limit surface is $C^{1}$. Notice that the eigenvalue $\frac{1}{8}$ of $\mathbf{S}$ has multiplicity 6 . Hence it is impossible to choose $x_{3}$ such that the condition $\left|\lambda_{3}\right|<\left|\lambda_{1}\right|^{2}=\left(\frac{1}{4}\right)^{2}$ for $C^{2}$ smoothness in Corollary 1 is fulfilled. In the following we choose $x_{3}=$ $1 / 2+1 / 16=9 / 16$.

Let $\triangle_{1}$ be the tetrahedron with vertices

$$
v_{0}^{0}=[0,0,1]^{T}, v_{1}^{0}=[1,0,0]^{T}, v_{2}^{0}=\left[-\frac{1}{2}, \frac{\sqrt{3}}{2}, 0\right]^{T}, v_{3}^{0}=\left[-\frac{1}{2},-\frac{\sqrt{3}}{2}, 0\right]^{T},
$$

as shown on the left of Fig. 15. Again we use these vertices as the first columns of the control vectors.

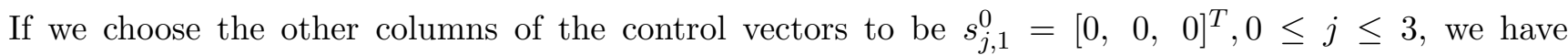
$v_{j}^{1}, j=0,1, \cdots, 10$, by one $S_{3}^{2}$-subdivision iteration step, as shown in the middle of Fig. 15 . The limit surface is displayed on the left of Fig. 15.

\section{Shape control of subdivision surfaces}

In this section, we first demonstrate that variation of the shape control parameters can change the shape of subdivision surfaces dramatically. We then give a preliminary result concerning the choices of the shape control parameters. 

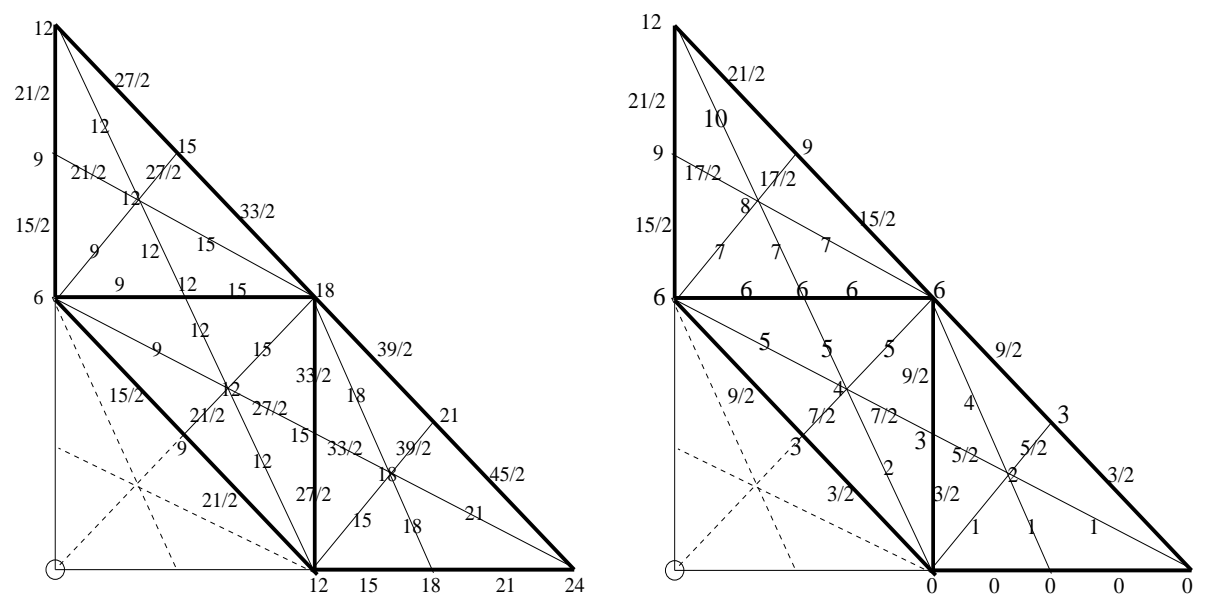

Figure 14: Bézier coefficients of $\frac{\partial \psi_{1}}{\partial v}$ (on left) and $\frac{\partial \psi_{2}}{\partial v}$ (on right) when $K=3$
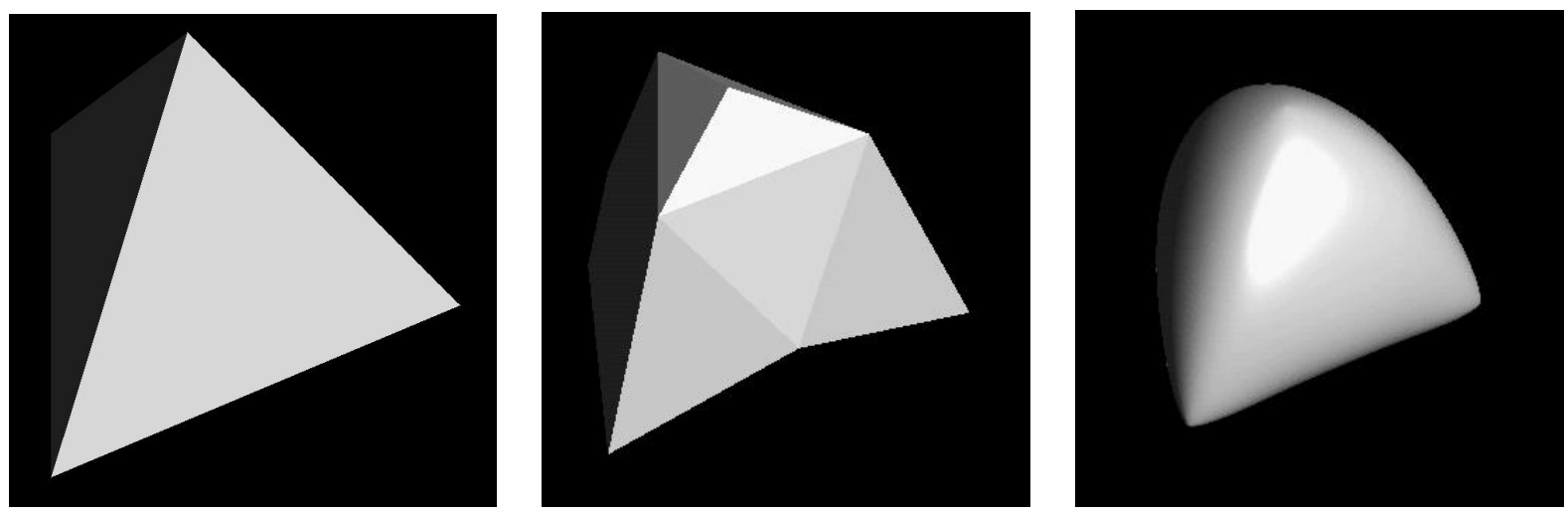

Figure 15: Initial closed surface (tetrahedron, on left), finer surface (in middle) after one iteration with shape control parameters $s_{j, 1}^{0}=[0,0,0]^{T}, 0 \leq j \leq 3$, limit surface (on right), by $S_{3}^{2}$-subdivision
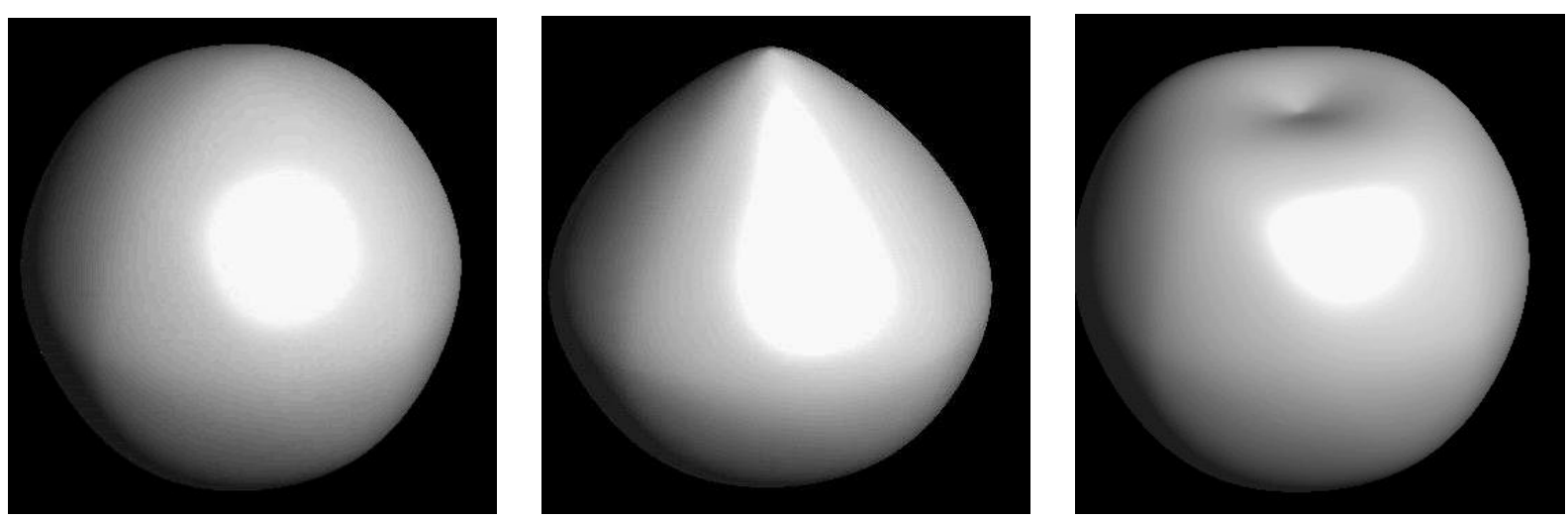

Figure 16: $S_{3}^{2}$-subdivision surfaces with same control vertices but with different shape control parameters $s_{0,1}^{0}=$ $[0,0,0]^{T}$ (on left), $s_{0,1}^{0}=[0,0,1]^{T}$ (in middle) and with $s_{0,1}^{0}=[0,0,-1]^{T}$ (on right) 

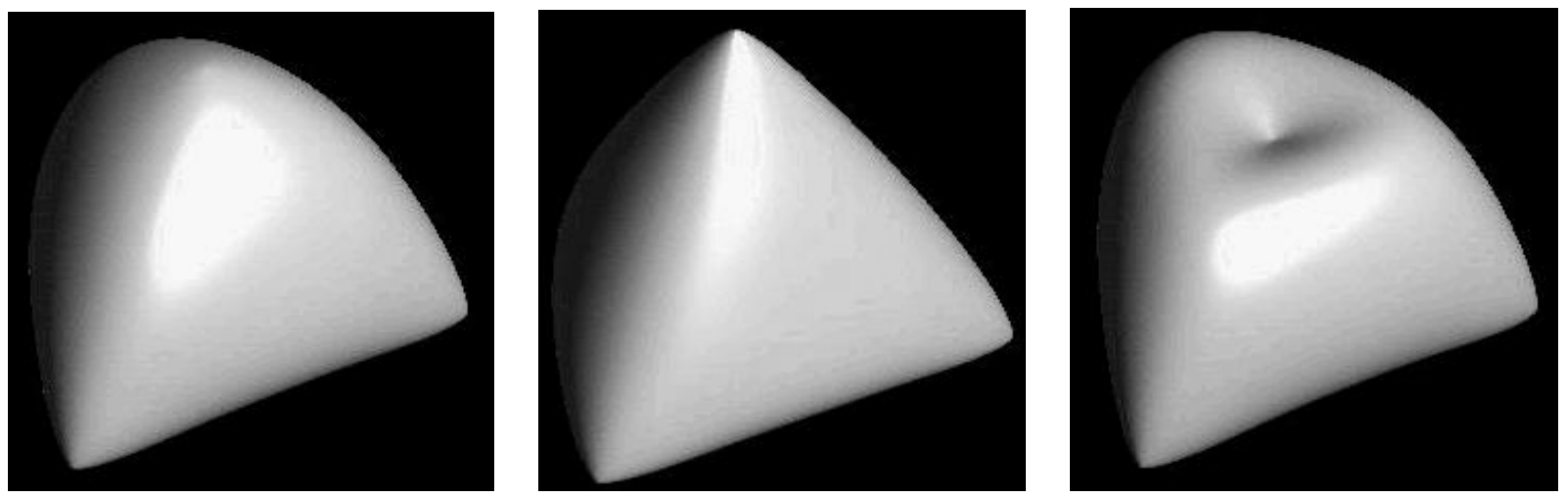

Figure 17: $S_{3}^{2}$-subdivision surfaces with same control vertices but with different shape control parameters $s_{0,1}^{0}=$ $[0,0,0]^{T}$ (on left), $s_{0,1}^{0}=[0,0,1]^{T}$ (in middle) and with $s_{0,1}^{0}=[0,0,-1]^{T}$ (on right)

\subsection{Shape control of surfaces}

Let us again consider the octahedron $\triangle_{0}$. With $x_{4}=9 / 16$, if we set the shape control parameters $s_{j, 1}^{0}$ to be $[0,0,0]^{T}$ for all $0 \leq j \leq 5$, then we have the subdivision surface shown in the first figure (from the left) in Fig. 16. However, by choosing only one shape control parameter $s_{0,1}^{0}$ to be $[0,0,1]^{T}$ and $[0,0,-1]^{T}$, we have the second and third subdivision surfaces shown in Fig. 16, respectively.

Similarly, for the tetrahedron $\triangle_{1}$, with $x_{3}=9 / 16$, if we choose the shape control parameter $s_{j, 1}^{0}=$ $\left[\begin{array}{lll}0, & 0, & 0\end{array}\right]^{T}$, for all $0 \leq j \leq 5$, the subdivision surface is shown in the first picture from the left. By replacing only one shape control parameter $s_{0,1}^{0}$ by $[0,0,1]^{T}$ and $[0,0,-1]^{T}$, we have the subdivision surfaces shown in the second and third pictures in Fig. 17, respectively. An advantage of the matrixvalued subdivision schemes is that surface shapes can be re-designed easily by adjusting the shape control parameters.

\subsection{Choices of shape control parameters}

In this subsection we give a preliminary result on the choices of the shape control parameters $s_{\mathbf{j}, \ell}^{0}=$ : $\left[x_{\mathbf{j}, \ell}, y_{\mathbf{j}, \ell}, z_{\mathbf{j}, \ell}\right]^{T}, \ell=1, \cdots, r-1$. We derive that the shape control parameters should be related to the vectors for the sum rule order of the subdivision mask. In the following, for the simplicity of our presentation, we just consider the case $A=2 I_{2}$, though our result is valid for the general dilation matrix $A$.

Suppose $\Phi=\left[\phi_{0}, \cdots, \phi_{r-1}\right]^{T}$ is a compactly supported refinable function vector on $\mathbb{R}^{2}$ satisfying the refinement equation (2.1) for some finite sequence $\left\{P_{\mathbf{k}}\right\}$ of $r \times r$ matrices with $A=2 I_{2}$. Let

$$
P(\omega):=\frac{1}{4} \sum_{\mathbf{k} \in \mathbf{Z}^{2}} P_{\mathbf{k}} e^{-i \mathbf{k} \omega}
$$

be the two-scale symbol of $\Phi$. Then $P$ is said to possess the property of the sum rule of order $m$, if there exist $1 \times r$ vectors $\mathbf{w}_{\alpha},|\alpha|<m$ with $\mathbf{w}_{0} \neq \mathbf{0}$ such that for all $|\beta|<m$,

$$
\sum_{0 \leq \gamma \leq \beta}\left(\begin{array}{l}
\beta \\
\gamma
\end{array}\right)(2 i)^{|\gamma-\beta|} \mathbf{w}_{\gamma} D^{\beta-\gamma} P\left(\eta_{0}\right)= \begin{cases}2^{-|\beta|} \mathbf{w}_{\beta}, & \text { when } \eta_{0}=(0,0) \\
\mathbf{0}, & \text { when } \eta_{0}=(\pi, 0),(0, \pi),(\pi, \pi) .\end{cases}
$$

Here for multi-indices $\gamma=\left(\gamma_{1}, \gamma_{2}\right), \beta=\left(\beta_{1}, \beta_{2}\right) \in \mathbb{Z}_{+}^{2}$, the standard notations $|\gamma|=\gamma_{1}+\gamma_{2},\left(\begin{array}{l}\beta \\ \gamma\end{array}\right)=$ 
$\left(\begin{array}{c}\beta_{1} \\ \gamma_{1}\end{array}\right)\left(\begin{array}{c}\beta_{2} \\ \gamma_{2}\end{array}\right), D^{\gamma}=\frac{\partial^{\gamma_{1}+\gamma_{2}}}{\partial x^{\gamma_{1}} \partial y^{\gamma_{2}}}$ are used. It follows from (5.1) that

$$
\mathbf{x}^{\alpha}=\sum_{\mathbf{k}}\left\{\sum_{\beta \leq \alpha}\left(\begin{array}{c}
\alpha \\
\beta
\end{array}\right) \mathbf{k}^{\alpha-\beta} \mathbf{w}_{\beta}\right\} \Phi(\mathbf{x}-\mathbf{k}), \quad \mathbf{x} \in \mathbb{R}^{2},|\alpha|<m
$$

and hence, that $P$ satisfies the sum rule of order $m$ implies that all polynomials of total degree $m-1$ can be reproduced locally by integer translates of $\Phi$ (see the survey paper [16] and the references therein). In particular, if the two-scale symbol $P$ of $\Phi$ satisfies the sum rule of order (at least) 1 , meaning that there exists $\mathbf{w}_{0}=\left[w_{0,0}, w_{0,1}, \cdots, w_{0, r-1}\right] \neq \mathbf{0}$ such that

$$
\mathbf{w}_{0} P(0,0)=\mathbf{w}_{0}, \quad \mathbf{w}_{0} P(\pi, 0)=\mathbf{w}_{0} P(0, \pi)=\mathbf{w}_{0} P(\pi, \pi)=\mathbf{0}
$$

then $\Phi$ satisfies (2.2) with $\mathbf{w}=\mathbf{w}_{0}$. Furthermore, if $P$ satisfies the sum rule of order at least 2 with $\mathbf{w}_{0}, \mathbf{w}_{(1,0)}, \mathbf{w}_{(0,1)}, w_{0,0}=1$, say, then under the assumption $\Phi(x, y)=\Phi(y, x)$, the vectors $\mathbf{w}_{(1,0)}=$ $\left[w_{(1,0), 0}, \cdots, w_{(1,0), r-1}\right]$ and $\mathbf{w}_{(0,1)}=\left[w_{(0,1), 0}, \cdots, w_{(0,1), r-1}\right]$ in (5.1) or $(5.2)$ can be chosen to satisfy

$$
\mathbf{w}_{(1,0)}=\mathbf{w}_{(0,1)}
$$

In the following discussion, we assume that (5.4) holds.

Let the control vertices in $\mathbb{R}^{3}$ be given by

$$
v_{\mathbf{j}}^{0}=\left[j_{1}, j_{2}, z_{\mathbf{j}}\right]^{T}, \quad \mathbf{j}=\left(j_{1}, j_{2}\right) \in \mathbb{Z}^{2},
$$

for some $z_{\mathbf{j}} \in \mathbb{R}$. In the following, we will show that the shape control parameters $s_{\mathbf{j}, 1}^{0}, \cdots, s_{\mathbf{j}, r-1}^{0}($ with the first two components to be labelled by $x_{\mathbf{j}, 1}^{0}, \cdots, x_{\mathbf{j}, r-1}^{0}$ and $y_{\mathbf{j}, 1}^{0}, \cdots, y_{\mathbf{j}, r-1}^{0}$, respectively) can be chosen such that the subdivision surface is

$$
\begin{aligned}
& \sum_{\mathbf{k}} v_{\mathbf{j}}^{0} \phi_{0}(\mathbf{x}-\mathbf{j})+\sum_{\mathbf{k}}\left(s_{\mathbf{j}, 1}^{0} \phi_{1}(\mathbf{x}-\mathbf{j})+\cdots+s_{\mathbf{j}, r-1}^{0} \phi_{r-1}(\mathbf{x}-\mathbf{j})\right) \\
& =: \sum_{\mathbf{j}} \mathbf{v}_{\mathbf{j}}^{0} \Phi(\mathbf{x}-\mathbf{j})=[x, y, z(x, y)]^{T},
\end{aligned}
$$

for some function $z(x, y)$. In particular,

$$
\sum_{\mathbf{j}}\left[j_{1}, x_{\mathbf{j}, 1}, \cdots, x_{\mathbf{j}, r-1}\right] \Phi(\mathbf{x}-\mathbf{j})=x, \quad \sum_{\mathbf{j}}\left[j_{2}, y_{\mathbf{j}, 1}, \cdots, y_{\mathbf{j}, r-1}\right] \Phi(\mathbf{x}-\mathbf{j})=y .
$$

For this purpose, since the property of the sum rule of order 2 implies the preservation of linear polynomials, we observe that

$$
\sum_{\mathbf{j}}\left(\mathbf{w}_{(1,0)}+j_{1} \mathbf{w}_{0}\right) \Phi(\mathbf{x}-\mathbf{j})=x, \quad \sum_{\mathbf{j}}\left(\mathbf{w}_{(1,0)}+j_{2} \mathbf{w}_{0}\right) \Phi(\mathbf{x}-\mathbf{j})=y .
$$

Therefore, by equalities (5.5) and (5.6), we may choose $x_{\mathbf{j}, 1}, \cdots, x_{\mathbf{j}, r-1}$ and $y_{\mathbf{j}, 1}, \cdots, y_{\mathbf{j}, r-1}$ to satisfy

$$
\left[j_{1}, x_{\mathbf{j}, 1}, \cdots, x_{\mathbf{j}, r-1}\right]=\mathbf{w}_{(1,0)}+j_{1} \mathbf{w}_{0}, \quad\left[j_{2}, y_{\mathbf{j}, 1}, \cdots, y_{\mathbf{j}, r-1}\right]=\mathbf{w}_{(0,1)}+j_{2} \mathbf{w}_{0}=\mathbf{w}_{(1,0)}+j_{2} \mathbf{w}_{0}
$$

or

$$
\begin{aligned}
& {\left[x_{\mathbf{j}, 1}, \cdots, x_{\mathbf{j}, r-1}\right]=\left[w_{(1,0), 1}, \cdots, w_{(1,0), r-1}\right]+j_{1}\left[w_{0,1}, \cdots, w_{0, r-1}\right]} \\
& {\left[y_{\mathbf{j}, 1}, \cdots, y_{\mathbf{j}, r-1}\right]=\left[w_{(1,0), 1}, \cdots, w_{(1,0), r-1}\right]+j_{2}\left[w_{0,1}, \cdots, w_{0, r-1}\right]}
\end{aligned}
$$


Based on the above observation, for control vertices $v_{\mathbf{j}}^{0}=:\left[x_{\mathbf{j}}, y_{\mathbf{j}}, z_{\mathbf{j}}\right]^{T}$ in general, it is perhaps advisable, at least for an initial attempt, to select shape control parameters $s_{\mathbf{j}, \ell}^{0}=\left[x_{\mathbf{j}, \ell}, y_{\mathbf{j}, \ell}, z_{\mathbf{j}, \ell}\right]^{T}$ with

$$
\begin{aligned}
& {\left[x_{\mathbf{j}, 1}, \cdots, x_{\mathbf{j}, r-1}\right]=\left[w_{(1,0), 1}, \cdots, w_{(1,0), r-1}\right]+x_{\mathbf{j}}\left[w_{0,1}, \cdots, w_{0, r-1}\right],} \\
& {\left[y_{\mathbf{j}, 1}, \cdots, y_{\mathbf{j}, r-1}\right]=\left[w_{(1,0), 1}, \cdots, w_{(1,0), r-1}\right]+y_{\mathbf{j}}\left[w_{0,1}, \cdots, w_{0, r-1}\right],} \\
& {\left[z_{\mathbf{j}, 1}, \cdots, z_{\mathbf{j}, r-1}\right]=\left[w_{(1,0), 1}, \cdots, w_{(1,0), r-1}\right]+z_{\mathbf{j}}\left[w_{0,1}, \cdots, w_{0, r-1}\right] .}
\end{aligned}
$$

The problem on how to choose the shape control parameters "optimally" deserves further investigations.

Remark 2 Let $U$ be a nonsingular $2 \times 2$ constant matrix. Since $U \Phi^{b}$ is also refinable with the refinement mask $\left\{U P_{\mathbf{k}} U^{-1}\right\}$, one could use $\left\{U P_{\mathbf{k}} U^{-1}\right\}$ for the local averaging rules. More precisely, for local averaging rules, one could use the templates in Fig. 4 and Fig. 7 with $B, C, D, P_{0,0}$ and $Q_{0, K}$ replaced by $U B U^{-1}, U C U^{-1}, U D U^{-1}, U P_{0,0} U^{-1}$ and $U Q_{0, K} U^{-1}$, respectively. The characteristic map is still regular and injective. The subdivision surface is also in $C^{1}$ for $K=3$, and $C^{1}$ or $C^{2}$ for $K=4$, with the same choices of $x_{3}, x_{4}$. However, the shape of the subdivision surfaces could be different. The problem on an "optimal" choice of $U$ deserves further investigations. The choice of

$$
U=\left[\begin{array}{cc}
1 & 1 \\
t_{1} & t_{2}
\end{array}\right],
$$

where $t_{1}$ and $t_{2}$ are real numbers with $t_{1} \neq t_{2}$, is of particular interest to us since for this choice of $U$, $\mathbf{w}_{0}=[1,0]$ is the vector for which (5.3) holds when $P$ is replaced by the two-scale symbol corresponding to the mask $\left\{U P_{\mathbf{k}} U^{-1}\right\}$. For a vector subdivision scheme with $\mathbf{w}_{0}=[1,0], \mathbf{w}_{(1,0)}=\mathbf{w}_{(0,1)}=[0,0]$, we could set the shape control parameters to be $\mathbf{0}$ by the above discussion. In the case $\mathbf{w}_{0}=[1,0]$, the vector subdivision scheme is affine-invariant in the sense that it produces the same surfaces (up to some shift) with initial control vectors $\left\{\left[v_{k}^{0}, \mathbf{0}\right]\right\}_{k}$ and $\left\{\left[a v_{k}^{0}+v, \mathbf{0}\right]\right\}_{k}$, where $a>0$ and $v \in \mathbb{R}^{3}$.

\section{References}

[1] A.A. Ball and J.T. Storry, Conditions for tangent plane continuity of recursively generated B-splines, ACM Trans. Graphics 7(1988) 83-102.

[2] E. Catmull and J. Clark, Recursively generated B-splines surfaces on arbitrary topological meshes, Comput. Aided Design 10 (1978) 350-355.

[3] A.S. Cavaretta, W. Dahmen, and C.A. Micchelli, Stationary Subdivision, Amer. Math. Soc. Memoirs of AMS, \#93, 1991.

[4] M. Charina and C. Conti, Convergence of multivariate non-stationary vector subdivision schemes, Appl. Numer. Anal. 49 (2004) 43-354.

[5] C.K. Chui, Multivariate Splines, NSF-CBMS Series \#54, SIAM Publ., Philadelphia, 1988.

[6] C.K. Chui and Q.T. Jiang, Surface subdivision schemes generated by refinable bivariate spline function vectors, Appl. Comput. Harmonic Anal. 15 (2003) 147-162.

[7] C.K. Chui and Q.T. Jiang, Refinable bivariate $C^{2}$-splines for multi-level data representation and surface display, Math Comput. 74 (2005) 1369-1390. 
[8] A. Cohen, K. Gröchenig, and L. F. Villemoes, Regularity of multivariate refinable functions, Constr. Approx. 15 (1999) 241-255.

[9] D.W.H. Doo and M.A. Sabin, Analysis of the behaviour of recursive division surfaces near extraordinary points, Comput. Aided Design 10 (1978) 356-360.

[10] N. Dyn, D. Levin, and J.A. Gregory, A butterfly subdivision scheme for surface interpolation with tension control, ACM Trans. Graphics 2 (1990) 160-169.

[11] A. Habib and J. Warren, Edge and vetex insertion for a class of subdivision surfaces, Comput. Aided Geom. Design 16 (1999) 223-247.

[12] B. Han and R.Q. Jia, Quincunx fundamental refinable functions and quincunx biorthogonal wavelets, Math Comput. 71 (2002) 165-196.

[13] B. Han, T. Yu, and B. Piper, Multivariate refinable Hermite interpolants, Math Comput. 73 (2004) 1913-1935.

[14] I.P. Ivrissimtzis, N.A. Dodgson, M.F. Hassan, and M.A. Sabin, On the geometry of recursive subdivision, Internat. J. Shape Modeling 8 (2002), 23-42.

[15] R.Q. Jia, Characterization of smoothness of multivariate refinable functions in Sobolev spaces, Trans. Amer. Math. Soc. 351 (1999) 4089-4112.

[16] R.Q. Jia, and Q.T. Jiang, Approximation power of refinable vectors of functions, in: Wavelet analysis and applications, AMS/IP Stud. Adv. Math., Vol. 25, Amer. Math. Soc., Providence, RI, 2002, pp. 155-178.

[17] R.Q. Jia and Q.T. Jiang, Spectral analysis of transition operators and its applications to smoothness analysis of wavelets, SIAM J. Matrix Anal. Appl. 24 (2003) 1071-1109.

[18] R.Q. Jia, S.D. Riemenschneider, and D.X. Zhou, Vector subdivision schemes and multiple wavelets, Math. Comp. 67 (1998) 1533-1563.

[19] Q.T. Jiang, Multivariate matrix refinable functions with arbitrary matrix dilation, Trans. Amer. Math. Soc. 351 (1999) 2407-2438.

[20] Q.T. Jiang and P. Oswald, Triangular $\sqrt{3}$-subdivision schemes: the regular case, J. Comput. Appl. Math. 156 (2003) 47-75.

[21] Q.T. Jiang, P. Oswald, and S.D. Riemenschneider, $\sqrt{3}$-subdivision schemes: maximal sum rule orders, Constr. Approx. 19 (2003) 437-463.

[22] L. Kobbelt, $\sqrt{3}$-subdivision, SIGGRAPH Computer Graphics Proceedings, 2000, pp. 103-112.

[23] U. Labsik and G. Greiner, Interpolatory $\sqrt{3}$-subdivision, Computer Graphics Forum 19 (2000) 131138.

[24] G.Q. Li, W.Y. Ma, and H.J. Bao, $\sqrt{2}$ subdivision for quadrilateral meshes, Visual Computer 20 (2004) 180-189. 
[25] C. Loop, Smooth subdivision surfaces based on triangles, Master's Thesis, University of Utah, Department of Mathematics, Salt Lake City, 1987.

[26] C.A. Micchelli and T. Sauer, On vector subdivision, Math. Z. 229 (1998) 621-674.

[27] P. Oswald, Designing composite triangular subdivision schemes, Comput. Aided Geom. Design 22 (2005) 659-679.

[28] P. Oswald and P. Schröder, Composite primal/dual $\sqrt{3}$-subdivision schemes, Comput. Aided Geom. Design 20 (2003) 135-164.

[29] J. Peters and U. Reif, The simplest subdivision scheme for smoothing polyhedra, ACM Trans. Graphics 16 (1997) 34-73.

[30] J. Peters and U. Reif, Analysis of algorithms generalizing $B$-spline subdivision, SIAM J. Numer. Anal. 35 (1998) 728-748.

[31] H. Prautzsch, Analysis of $C^{k}$-subdivision surfaces at extraordinary points, preprint 04/98, Fakultät für Informatik, Universität Karlsuhe, Germany, 1998. (available at http://i33www.ira.uka.de)

[32] H. Prautzsch, Freeform splines, Comput. Aided Geom. Design 14 (1997) 201-206.

[33] H. Prautzsch and G. Umlauf, A $G^{2}$-subdivision algorithm, In: Geometric Modelling, G. Farin, H. Bieri, G. Brunnet, T. DeRose (eds.), Computing Suppl. 13, Springer Verlag, N.Y., 1998, pp. $217-224$.

[34] H. Prautzsch and G. Umlauf, A $G^{1}$ and a $G^{2}$ subdivision schemes for triangular nets, Int. J. Shape Modelling 6 (2000) 21-35.

[35] U. Reif, A unified approach to subdivision algorithms near extraordinary vertices, Comput. Aided Geom. Design 21 (1995) 153-174.

[36] U. Reif, TURBS-topologically unrestricted rational B-splines, Constr. Approx. 14 (1998) 57-77.

[37] J.E. Schweitzer, Analysis and application of subdivision surfaces, Ph.D. Thesis, University of Washington, Dept. of Computer Science and Engineering, Seattle, 1996.

[38] Z.W. Shen, Refinable function vectors, SIAM J. Math. Anal. 29 (1998) 235-250.

[39] G. Umlauf, Analyzing the characteristic map of triangular subdivision schemes, Constr. Approx. 16 (2000) 145-155.

[40] L. Velho, Quasi 4-8 subdivision, Comput. Aided Geom. Design 18 (2001) 345-357.

[41] L. Velho and D. Zorin, 4-8 subdivision, Comput. Aided Geom. Design 18 (2001) 397-427.

[42] D. Zorin, A method for analysis of $C^{1}$-continuity of subdivision surfaces, SIAM J. Numer. Anal. 37 (2000) 1677-1708.

[43] D. Zorin, Smoothness of subdivision on irregular meshes, Constr. Approx. 16 (2000) 359-397. 